\title{
Monitoring Gas Retention and Slurry Transport During the Transfer of Waste from Tank 241-C-106 to Tank 241-AY-102
}

\author{
C. W. Stewart \\ F. F. Erian \\ P. A. Meyer \\ K. P. Recknagle \\ W. B. Gregory \\ Z. I. Antoniak \\ D. M. Pfund
}

July 1997

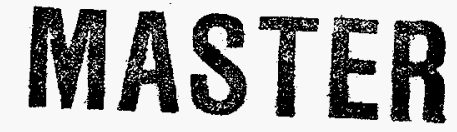

DASTROUTON OF THIS DOCUNENT IS UW MARED

Prepared for

the U.S. Department of Energy

under Contract DE-AC06-76RLO 1830

Pacific Northwest National Laboratory

Richland, Washington 


\section{DISCLAIMER}

This report was prepared as an account of work sponsored by an agency of the United States Government. Neither the United States Government nor any agency thereof, nor any of their employees, make any warranty, express or implied, or assumes any legal liability or responsibility for the accuracy, completeness, or usefulness of any information, apparatus, product, or process disclosed, or represents that its use would not infringe privately owned rights. Reference herein to any specific commercial product, process, or service by trade name, trademark, manufacturer, or otherwise does not necessarily constitute or imply its endorsement, recommendation, or favoring by the United States Government or any agency thereof. The views and opinions of authors expressed herein do not necessarily state or reflect those of the United States Government or any agency thereof. 


\section{DISClamier}

Portions of this doement may be illegible in electronic image products. Irrages are produced from the best available original document. 


\section{Summary}

\section{Gas Retention}

The retained gas volume can be estimated by several methods. All of these methods have significant uncertainties, but together they form a preponderance of evidence that describes the gas retention behavior of the tank. The methods are

1. An increase in nonconvective layer thickness

2. A waste surface level rise (surface level effect [SLE] model)

3. The barometric pressure effect (BPE model)

4. Direct void measurement

5. The consequences of the transfer process.

The nonconvective layer thickness can be determined with sufficient accuracy to describe the overall waste configuration by means of temperature profiles or densitometer indications. However, the presence of a nonconvective layer does not necessarily indicate significant gas retention, and small changes in layer thickness that could quantify gas retention cannot be detected reliably with the methods available. The primary value of this measurement is in establishing the actual "fluffing factor" for thermal calculations.

Surface level rise is not a useful measure of gas retention in Tank 241-C-106 (C-106) since the waste level fluctuates with regular makeup water additions. In Tank 241-AY-102 (AY-102) with the existing ventilation system it should be possible to determine the gas retention rate within a $30-60 \%$ uncertainty from the surface level rise, should a significant rise be observed. The planned ventilation system upgrades in AY-102 will greatly reduce the exhaust flow and the headspace humidity, and the evaporation rate should be significantly lower when transfers begin. This could reduce the uncertainty in gas retention rate estimates to around $\pm 10 \%$.

The barometric pressure response in C-106 has been difficult to assess because of the scatter in the Enraf ${ }^{\circledast}$ level gauge analog signal output compared with the small level changes. Enraf level data for a two-week period in November 1995, during which large barometric pressure swings occurred, indicated a void fraction of $7.5 \pm 5 \%$. The raw digital output will soon be recorded at about an order of magnitude better precision. This should provide a clean correlation of surface level and barometric pressure, and a gas volume fraction of $\sim 5 \%$ should be measurable with a relative uncertainty on the order of $50 \%$ with nominal barometric pressure fluctuations.

The BPE method has a somewhat higher uncertainty in AY-102 after transfer than in C-106 because of the increased waste depth and resulting higher hydrostatic pressure. Using the analog output of the Enraf level gauge, the void fraction must reach about $10 \%$ before the gas volume can be quantified with any accuracy. However, void fractions of a few percent should be detectable during the larger barometric pressure swings. The BPE method will serve mainly to confirm rather than initially detect significant gas retention in AY-102.

It is not practical to attempt to measure void fraction in C-106 with the void fraction instrument (VFI) or retained gas sampler (RGS), because there are few risers available in which to operate this equipment. In fact, there are no accessible risers available for the RGS, and the only risers available to the VFI are near the tank wall and would not be representative of the whole tank. 
Also, the radiation dose to the workers limits riser selection to those outside the pump pits. On the other hand, there are ample risers available in AY-102, and the waste will be relatively uniform, so a local void measurement would have a high probability of being representative of the entire tank. However, neither the VFI nor the R.GS should be used until after complete transfer and then only after there are other indications of significant gas retention.

It is possible to estimate the gas volume in C-106 after the fact by measuring the gas volume released when the waste is disrupted by sluicing. But there is at least $\pm 50 \%$ uncertainty in how much sludge is actually disturbed. The resulting error in the void fraction could be as high as $100 \%$. However, at some point when the transfer is nearly complete, essentially all of the sludge will have been disturbed and the uncertainty in the calculated void fraction reduces to $\sim 40 \%$. Therefore, the gas released during sluicing can be taken as a fairly accurate indicator of the average void fraction in C-106 after most of the waste is transferred. It should not, however, be used as a quantitative calculation in the initial stages of the operation.

If AY-102 does not retain gas, the sum of the gas generation rates in both tanks prior to transfer should be approximately equal to the sum of release rates measured after transfer. If the post-transfer sum is less, one can deduce that AY-102 is retaining gas at a rate about equal to the difference. Assuming that each of the current and post-transfer generation rates is known to within $\pm 30 \%$, the retention rate estimated for AY-102 will be accurate only to within $\pm 50 \%$. This method should thus be considered only a qualitative indicator of gas retention at best.

\section{Slurry Handling}

Mobilization of sludge waste is achieved by sluicing. The sluicing strategy affects many of the process control operations. It is not realistic to expect that a uniform sludge layer will be removed from the waste cylinder considering the geometry and locations of the sluicing jet and the retrieval pump; a shallow incline, or grade, is required to assist in the retrieval process.

Creating the required grade means that sludge will be disturbed to a greater depth than planned in this transfer stage. That is, to remove the top two feet it may be necessary to disturb four or more feet near the pump. Thus a higher than expected heat load may be transferred in the first 2-ft stage because the concentration of heat-generating isotopes increases with sludge depth. Many of the heat transfer calculations may be influenced by the practical aspects of solids removal via the installed sluicing/retrieval geometry. Also, it will not be practical to determine the original gas content of $\mathrm{C}-106$ from the volume of gas released during sluicing until most of the waste has been removed.

An important process control device is the Micromotion mass flowmeter. Uncertainty calculations show that when using that instrument to monitor the amount of solids being transferred from C-106 to AY-102, accuracy is enhanced considerably by relying on the sluicing time if the solids concentration is high, $C_{v}>0.1$. At lower solids concentrations, $C_{v} \leq 0.1$, the volume flow rate of the solid particles is a more accurate measure of the solids being transported and should be used as the process control parameter.

The feasibility of monitoring in real time the heat load associated with the radioactive waste being transferred as a slurry from C-106 to AY-102 has been evaluated. Cesium-137 has a strong gamma emission and thus can be easily detected in the pipe during transport; however, determining the quantity of ${ }^{90} \mathrm{Sr}$ in the transfer line is much more difficult. Methods based on the measurement of the bremsstrahlung spectra, or a bremsstrahlung coincidence approach, are being developed. Acquiring the data is relatively easy, but relating those data to actual ${ }^{90} \mathrm{Sr}$ concentration requires a difficult calibration of the counting geometry versus the waste stream composition. 


\section{Data Management}

The data related to both C-106 and AY-102 that are necessary to compute potential changes in the conditions of each tank must be captured and made generally available for analysis. The data related to transfer of material from each tank to another must also be available for analysis to confirm safe operating conditions and to monitor the volume and types of material transferred. The data must be observable in near real time to support safe operations and to confirm transfer of material. The data must also be stored for later analysis by engineering staff so that heat load and flammable gas related issues can be evaluated. 



\section{Contents}

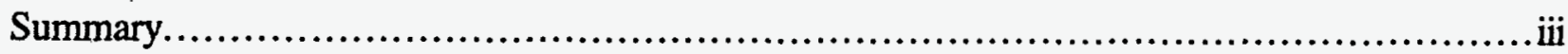

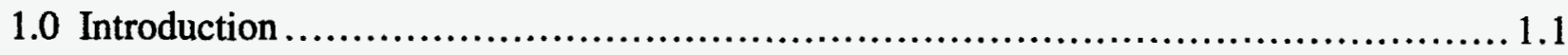

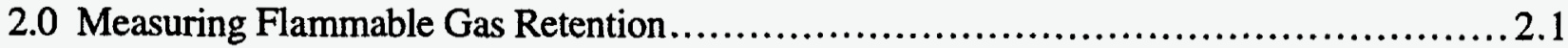

2.1 Determining Nonconvective Layer Thickness ............................... 2.1

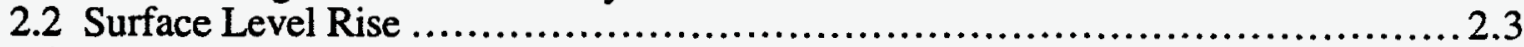

2.3 Barometric Pressure Response ................................................ 2.4

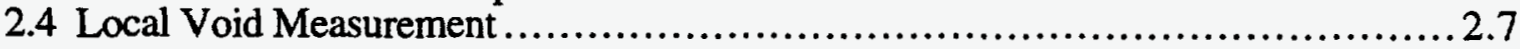

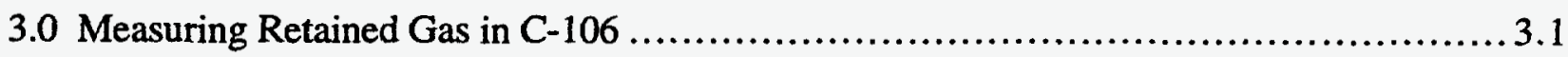

3.1 Barometric Pressure Response .................................................. 3.1

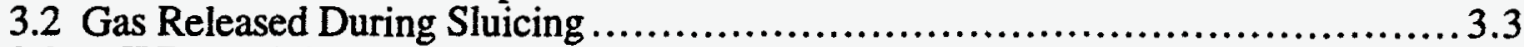

3.3 VFI/RGS Void Fraction Measurements ….................................. 3.4

4.0 Monitoring Gas Retention in AY-102 After Transfer ................................. 4.1

4.1 Barometric Pressure Response .............................................. 4.1

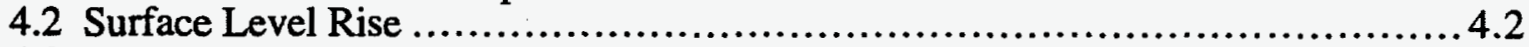

4.3 Conservation of Gas Generation in C-106 and AY-102 ........................ 4.3

4.4 VFI/RGS Void Fraction Measurements in AY-102 ........................... 4.4

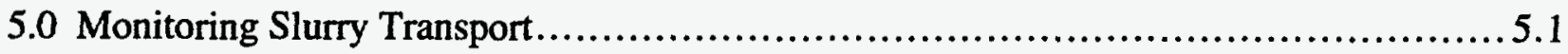

5.1 Sluicing Operations .............................................................

5.2 Operational Process Control Instrumentation ................................. 5.4

5.2.1 The Micromotion Instrument as a Process Control Device....................5.5

5.2.2 Monitoring Heat Load Transfer....................................... 5.9

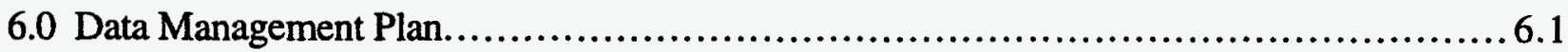

6.1 Data Format and Content ........................................................ 6.1

6.2 Data Handling........................................................................................ 6.1

6.3 Data Management Team ........................................................... 6.2

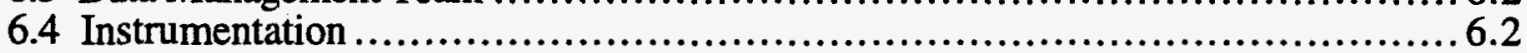

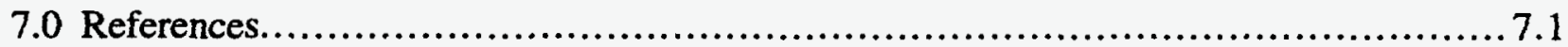




\section{Figures}

2.1 Waste Temperature Profiles in DSTs

2.2 Minimum Detectable Void Fraction, C-106 Before Transfer.............................. 2.6

2.3 Minimum Detectable Void Fraction, AY-102 After Transfer......................... 2.6

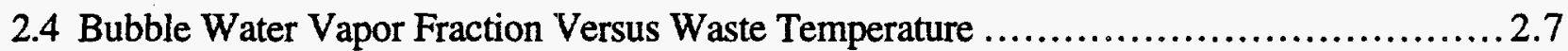

3.1 Enraf Surface Level History for C-106: January 8 and 9, 1997.......................2

3.2 Void Fraction Detectable via BPE in C-106 for Several Level Sensitivities.................. 3.2

4.1 Void Fraction Detectable via BPE in AY-102 for Several Level Sensitivities............... 4.2

4.2 Uncertainty in Gas Retention via the Surface Level Effect Model ......................4.3

5.1 Schematic of Sludge Cylinder with Sluicing Jet and Slurry Pump Locations................ 5.2

5.2 Sludge Removed when 2-ft Depth Is Reached at Pump End........................... 5.3

5.3 Sludge Removed Equivalent to 2-ft Uniform Slice, 50,000 $\mathrm{ft}^{3} \ldots \ldots \ldots \ldots \ldots \ldots \ldots \ldots \ldots . . \ldots . . . \ldots$

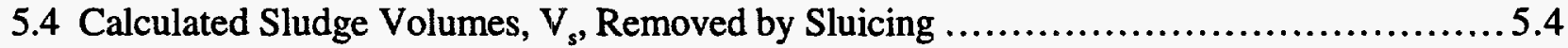

5.5 Parametric Contributions to Uncertainty in Sluicing Time ............................. 5.7

5.6 Overall Uncertainty in Sluicing Time ................................................ 5.7

5.7 Parametric Contributions to Uncertainty in the Solids Volume Flow Rate ................5.8

5.8 Overall Uncertainty in the Solids Volume Flow Rate .................................... 5.9 


\subsection{Introduction}

The waste in single-shell Tank 241-C-106 (C-106) is planned to be retrieved by sluicing and transferring it to double-shell Tank 241-AY-102 (AY-102) late in FY 1998 under Project $\mathrm{W}-320$. The objective of transferring waste from C-106 to AY-102 is to resolve the high-heat safety issue in C-106 and to demonstrate single-shell tank (SST) waste retrieval technology. Three major issues involved in the transfer are ensuring sufficient cooling and preventing flammable gas accumulation and release in AY-102 and monitoring and controlling the slurry transport process to accomplish these requirements. This report focuses on slurry transport and monitoring flammable gas retention. The cooling issue is covered in detail in the thermal evaluation by Sathyanayarana et al. (1997) and Sathyanarayana (1996). The entire flammable gas safety issue is discussed in a topical report by Pasamehmetoglu et al. (1997).

All radioactive waste generates flammable gases that are usually released continuously into the tank headspace at about the same rate as they are generated-where concentrations are kept low by passive or active ventilation (Wilkins et al. 1997). However, if the waste accumulates gas and the tank has a deep layer of supernatant liquid, there is a potential for a "rollover" that could create flammable concentrations in the headspace (Meyer et al. 1997). After transfer, AY-102 will have a relatively high gas generation rate as well as a deep supernatant layer. Therefore, although neither AY-102 nor C-106 has demonstrated significant gas retention, and none is expected after transfer, it is necessary to monitor gas retention so that any departure from expected behavior can be detected as soon as possible, and mitigation can be initiated.

The high heat load in C-106 requires adding water periodically to make up for evaporation and creates unacceptably high waste temperatures in the 50-year-old tank. The ventilation system in AY-102 will be enhanced specifically to handle the increased heat load of the C-106 waste (Farnworth 1997). But there is a concern that the waste will "fluff" following transfer to possibly twice its original volume. The increased volume acts as insulation and increases the waste temperatures. On the other hand, it is likely that some of the C-106 solids will dissolve in AY-102, and other experimental data suggest that the "fluffing factor" is less than 2 (Reynolds 1997). This uncertainty in the final waste configuration in AY-102 makes it very important to determine the actual amount of C-106 solids transferred at each stage of the process. It would also be desirable to be able to measure the corresponding heat load added to AY-102.

This report discusses methods for using the instrumentation available in both AY-102 and C-106 as well as some additional options for monitoring flammable gas retention and slurry transport. Section 2 describes methods for flammable gas retention, and Sections 3 and 4 discuss their application specifically to C-106 and AY-102, respectively. Section 5 is devoted to slurry transport monitoring, and Section 6 makes recommendations on managing the monitoring data. The reference list is given in Section 7. 


\subsection{Measuring Flammable Gas Retention}

The retained gas volume can be estimated using the following methods, individually and in combination:

1. Increase in nonconvective layer thickness: The layer of settled solids develops a yield stress and can potentially retain gas. The solids also suppress convection, so the layer is termed the nonconvective layer. The layer thickness can be determined from the temperature profiles or a densitometer bob. An increase in the thickness of this layer indicates a potential for gas accumulation.

2. Waste surface level rise: Gas accumulation in the nonconvective layer displaces the solids around it or displaces liquid from the pores between solid particles. In either case, the gas accumulation causes the waste level to rise. The gas retention rate can be calculated directly from the level rise rate corrected for evaporation and waste addition or removal.

3. Barometric pressure effect: Gas stored in the waste compresses and expands in response to changes in barometric pressure, causing the waste level to rise and fall. The gas volume can be estimated from the correlation of waste level changes and barometric pressure fluctuation.

4. Direct void measurement: The local gas volume fraction (void fraction) can be measured directly by the void fraction instrument (VFI) or the retained gas sampler (RGS). The total retained gas volume is calculated from the average of these local measurements (Stewart et al. 1996; Meyer et al. 1997; Shekarriz et al. 1997).

5. Consequences of the transfer process: The changes wrought in the waste by sluicing and transfer can be used to infer gas retention. These include determining the initial gas volume in C-106 from the volume released when the waste is disturbed by sluicing and inferring the retention rate in AY-102 by comparing the steady-state gas release in both tanks before and after transfer.

The combination of methods 1,2 , and 3 is the simplest and least costly and can be used for essentially continuous monitoring of changes in retained gas volume. Method 4, direct void measurement, is costly but much more accurate. Method 5 also provides indications if not quantitative values. These methods will be discussed in general in Sections 2.1-2.4 and specifically in terms of Tanks C-106 and AY-102 in Sections 2 and 3, respectively.

\subsection{Determining Nonconvective Layer Thickness}

The suppression of convection in the nonconvective layer creates a characteristic parabolic temperature profile from which the layer thickness can be determined. The layer height can also be measured directly by the ball rheometer or the Enraf ${ }^{\otimes}$ densitometer. Both methods can measure the nonconvective layer thickness with sufficient accuracy to describe the overall waste configuration, but neither is able to detect small changes that would indicate gas retention.

Figure 2.1 shows the temperature profiles in five of the double-shell tanks (DSTs) on the Flammable Gas Watch List. The top of the nonconvective layer is the elevation of the intersection of the parabolic portion of the profile and the uniform temperature of the convective layer above it. The figure indicates that these tanks have a nonconvective layer ranging from $-300 \mathrm{~cm}$ to almost $500 \mathrm{~cm}$ thick. The nonconvective layer in AY-102 following transfer is expected to be on the order of $200 \mathrm{~cm}$ thick. 
Where the parabolic profile is well developed it is usually possible to determine the thickness of the nonconvective layer to within half the distance between temperature measurements, provided that temperatures are recorded to a precision of at least $0.1^{\circ} \mathrm{F}$. In a typical multifunction instrument tree (MIT) with a thermocouple spacing of $30 \mathrm{~cm}$, the nonconvective layer depth can be measured to within $\pm 15 \mathrm{~cm}$. The MIT validation probe can provide temperature measurements about every $5 \mathrm{~cm}$ (typical runs measure every $10 \mathrm{~cm}$ ). With this close spacing of temperature measurements, the curvature of the profile at the convective-to-nonconvective transition limits the precision in the nonconvective layer depth. In the DSTs, the uncertainty is typically $\pm 8 \mathrm{~cm}$ (Stewart et al. 1996). This uncertainty is small enough to allow determination of the nonconvective layer depth and detection of level changes due to in-situ gas accumulation greater than about $30 \mathrm{~m}^{3}\left(1,000 \mathrm{ft}^{3}\right)$. This is equivalent to a void fraction of about 0.04 in $\mathrm{AY}-102$ after transfer.

An Enraf densitometer is planned for installation in AY-102 prior to sluicing. This instrument computes fluid density based on changes in the tension on a cable suspending a negatively buoyant plummet in the fluid. The principle of operation is exactly the same as the 16-lb tungsten ball rheometer that was deployed in the DSTs on the Flammable Gas Watch List (Stewart et al. 1996), except the densitometer plummet is much lighter. The densitometer provides accurate densities only in a liquid, i.e., where the yield stress is negligible. However, it will measure the height of the nonconvective layer to within $0.1 \mathrm{~mm}$ at a single location. This precision is sufficient to provide an alternate barometric pressure response calculation in addition to that derived from the waste surface level (see Section 2.3).

Erosion of the liquid-solid interface is a potential problem with the densitometer. Each time the densitometer is lowered to the interface, it can excavate a depression in the solids layer that could eventually compromise the accuracy of the nonconvective layer thickness measurement. To prevent this, the solids layer height should not be measured more than about once a month when

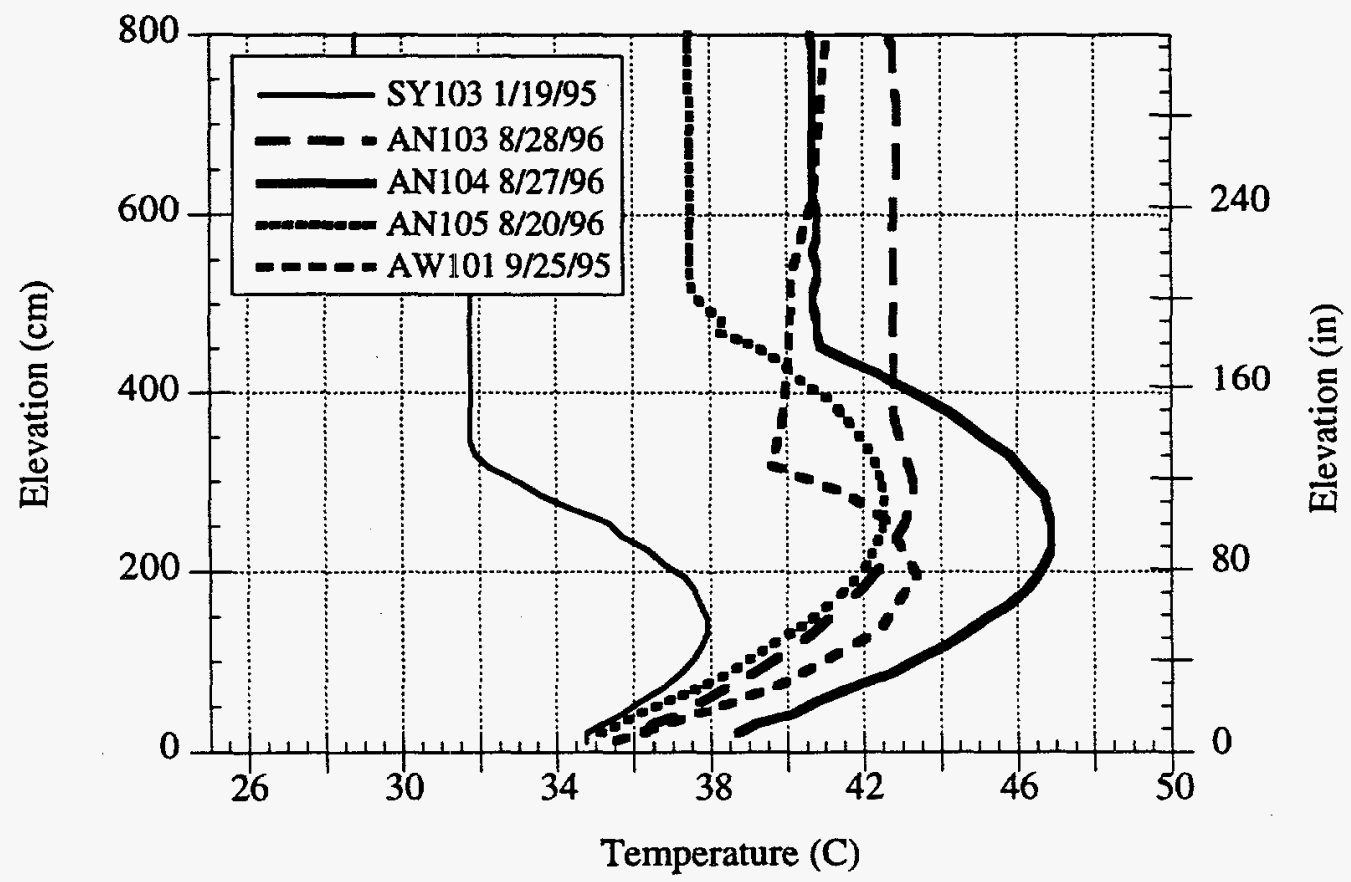

Figure 2.1. Waste Temperature Profiles in DSTs 
waste is not being transferred. In addition to the issue of surface erosion, there is some evidence that gas accumulates partially by expelling liquid as well as displacing solid particles. Due to both these effects, an increase in nonconvective layer thickness measured by the densitometer better represents a lower bound on gas retention than an actual measurement.

The nonconvective layer height in AY-102 could potentially be nonuniform because the incoming waste from C-106 will be discharged near the center of the tank. However, fluid dynamic simulations performed for a recent criticality study (Whyatt et al. 1997) predict a fairly uniform solids distribution. Accordingly, the average of the heights determined by the densitometer and the MIT validation probe temperature profile can be assumed to represent the nonconvective layer height throughout the entire tank.

\subsection{Surface Level Rise}

A rise in waste level is a measure of the amount of gas accumulating in the waste (if any). The in-situ volume of the retained gas is simply the level change times the tank area. This is called the surface level effect (SLE) model. However, other factors that affect the surface level, such as evaporation and net waste addition, must also be included. At any instant the rate of gas retention is described by

$$
\dot{\mathrm{V}}=\left[\dot{\mathrm{L}}_{\mathrm{W}}+\dot{\mathrm{L}}_{\mathrm{E}}-\dot{\mathrm{L}}_{\mathrm{A}}\right] \mathrm{A}
$$

where $\dot{V}$ is the gas volume retention rate, $\dot{L}_{W}$ is the measured surface level rise rate, $\dot{L}_{E}$ is the linear evaporation rate, $\dot{L}_{A}$ is the net linear rate of waste addition, and $A$ is the tank surface area. The cumulative in-situ gas retention is estimated from the surface level rise by integrating Eq. (2.1) over a given time period to yield

$$
\Delta \mathrm{V}_{\mathrm{G}}=\mathrm{A}\left[\Delta \mathrm{L}_{\mathrm{W}}+\Delta \mathrm{L}_{\mathrm{E}}-\Delta \mathrm{L}_{\mathrm{A}}\right]
$$

where $\Delta \mathrm{V}_{\mathrm{G}}$ is the change in in-situ gas volume, $\Delta \mathrm{L}_{\mathrm{W}}$ is the measured surface level change, $\Delta \mathrm{L}_{\mathrm{E}}$ is the level drop due to evaporation, and $\Delta \mathrm{L}_{\mathrm{A}}$ is the level rise due to net waste addition.

Waste level in both tanks is measured by an Enraf buoyancy gauge, which is capable of resolving level changes to $0.1 \mathrm{~cm}$ or less. No crust exists in C-106 nor is one expected to form in AY -102 , so the fractional uncertainty of the level measurement is expected to be negligible. The largest uncertainty in computing the gas volume via surface level rise is anticipated to be the error in estimating the evaporation effect. The uncertainty in the gas retention can be derived from Eq. (2.2) as

$$
\left(\frac{\sigma_{\mathrm{V}}}{\Delta \mathrm{V}_{\mathrm{G}}}\right)^{2}=\mathrm{A}\left[\frac{\Delta \mathrm{L}_{\mathrm{W}}}{\Delta \mathrm{V}_{\mathrm{G}}}\left(\frac{\sigma_{\mathrm{L}}}{\Delta \mathrm{L}_{\mathrm{W}}}\right)\right]^{2}+\mathrm{A}\left[\frac{\Delta \mathrm{L}_{\mathrm{E}}}{\Delta \mathrm{V}_{\mathrm{G}}}\left(\frac{\sigma_{\mathrm{E}}}{\Delta \mathrm{L}_{\mathrm{E}}}\right)\right]^{2}+\mathrm{A}\left[\frac{\Delta \mathrm{L}_{\mathrm{A}}}{\Delta \mathrm{V}_{\mathrm{G}}}\left(\frac{\sigma_{\mathrm{A}}}{\Delta \mathrm{L}_{\mathrm{A}}}\right)\right]^{2}
$$

where $\sigma_{i}$ represents the standard error in each quantity.

The evaporation uncertainty dominates the right side of Eq. (2.3). Since the error in the level measurement is fixed, while the measured level change increases with time (assuming gas is being retained), the first term on the right side of Eq. (2.3) decreases with time. Likewise, waste addition and removal are expected to occur over a relatively short time, so the resulting net level 
change will be fixed for most of the gas growth period. Thus the last term on the right also decreases with time as the retained gas volume grows. Also, the relative uncertainties in the measured level and in the waste addition/removal are expected to be quite small. On the other hand, the amount of evaporation is not constant, and its uncertainty is expected to be relatively large.

Neglecting the two small and decreasing terms in Eq. (2.3) and assuming the gas retention and evaporation rates are approximately constant (for the purpose of estimating uncertainty) provides the following expression for the uncertainty in gas retention at relatively long times:

$$
\left(\frac{\sigma_{\mathrm{V}}}{\Delta \mathrm{V}_{\mathrm{G}}}\right)=\frac{\mathrm{AL}_{\mathrm{E}}}{\dot{\mathrm{V}}}\left(\frac{\sigma_{\mathrm{E}}}{\dot{\mathrm{L}}_{\mathrm{E}}}\right)
$$

The standard gas volume, $\hat{\mathrm{V}}_{\mathrm{G}}$, is derived from the in-situ volume by correcting to standard pressure and temperature:

$$
\Delta \hat{\mathrm{V}}_{\mathrm{G}}=\mathrm{P}_{\mathrm{G}} \Delta \mathrm{V}_{\mathrm{G}}
$$

where $P_{G}$ is the average effective pressure ratio of the stored gas. It corrects for both nonstandard pressure and temperature. It is defined as

$$
P_{G}=\frac{p_{G}}{\hat{p}} \frac{\hat{T}}{T_{G}}
$$

where $\hat{\mathrm{p}}$ is standard atmospheric pressure $(101,320 \mathrm{~Pa}), \hat{\mathrm{T}}$ is standard temperature $(288 \mathrm{~K})$, and $T_{G}$ is the average temperature of the stored gas. $\mathrm{p}_{\mathrm{G}}$ is the average pressure computed from the hydrostatic head at the midpoint of the waste where the gas is stored as

$$
p_{\mathrm{G}}=\hat{\mathrm{p}}+\rho_{\mathrm{SN}} \mathrm{gH}_{\mathrm{SN}}+\rho_{\mathrm{NC}} \mathrm{gH}_{\mathrm{NC}} / 2
$$

where $\rho_{S N}$ and $\rho_{N C}$ are the densities and $H_{S N}$ and $H_{N C}$ are the heights of the supernatant and nonconvective layer, respectively. Note that $\mathrm{H}_{S N}$ can be replaced with $\mathrm{L}_{\mathrm{W}}-\mathrm{H}_{\mathrm{NC}}$. The gas pressure is currently about $1.1 \mathrm{~atm}$ in C-106 and about $1.8 \mathrm{~atm}$ in AY-102.

\subsection{Barometric Pressure Response} by

The total in-situ gas volume can also be computed from the barometric pressure response

$$
\mathrm{V}_{\mathrm{G}}=-\mathrm{Ap}_{\mathrm{G}} \frac{\mathrm{dL}_{\mathrm{W}}}{\mathrm{dP}}
$$

where $\mathrm{p}_{\mathrm{G}}$ is defined by Eq. (2.7) above and $\mathrm{dL}_{\mathrm{w}} / \mathrm{dP}$ is the response of the waste surface level to barometric pressure changes. This technique for computing the retained gas volume is called the barometric pressure effect (BPE) model. A detailed derivation of this model is given in Meyer et al. (1997). The standard gas volume can be found from the in-situ volume via Eq. (2.5). 
The advantage of the barometric pressure response calculation is that it is not affected by evaporation or other cumulative uncertainty the way the surface level rise method is. However, it is subject to uncertainty in the gas pressure and to errors due to the limited range of barometric pressure fluctuations and the precision of the level instruments.

A minimum detectable volume can be derived based on a given gas volume uncertainty threshold and level measurement error. The barometric response in Eq. (2.8) can be rewritten in finite difference form as

$$
\mathrm{V}_{\mathrm{G}}=-\mathrm{Ap}_{\mathrm{G}} \frac{\dot{\Delta \mathrm{L}_{\mathrm{W}}}}{\Delta \mathrm{P}}
$$

where $\Delta \mathrm{L}_{\mathrm{W}}$ is the specific level change in response to a corresponding barometric pressure change of $\Delta \mathrm{P}^{(\mathrm{a})}$ Assuming the barometric pressure change and tank area are given (i.e., not subject to uncertainty), the relative uncertainty in the retained gas volume in Eq. (2.9) can be estimated by linear error propagation as

$$
\left(\frac{\sigma_{\mathrm{V}}}{\mathrm{V}_{\mathrm{G}}}\right)^{2}=\left(\frac{\sigma_{\mathrm{p}}}{\mathrm{p}_{\mathrm{G}}}\right)^{2}+\left(\frac{\Delta \mathrm{L}_{\min }}{\Delta \mathrm{L}_{\mathrm{W}}}\right)^{2}
$$

where higher order terms have been ignored. Also, the relative uncertainty in level response has been approximated by the instrument precision divided by the actual level change. The level change is related to the gas volume and barometric pressure change through Eq. (2.9). Solving Eq. (2.9) for $\Delta \mathrm{L}_{\mathrm{w}}$ and substituting the result in Eq. (2.10) yields

$$
\left(\frac{\sigma_{V}}{V_{G}}\right)^{2}=\left(\frac{\sigma_{p}}{p_{G}}\right)^{2}+\left(\frac{A P_{G} \Delta L_{\text {min }}}{V_{G} \Delta P}\right)^{2}
$$

We seek the minimum gas volume that can be determined with an acceptably low error with a given level measurement sensitivity, gas pressure, and range of barometric pressure fluctuation. This can be accomplished most easily by specifying the left side of Eq. (2.11) and solving for $V_{G}$, to yield

$$
\mathrm{V}_{\mathrm{MIN}}=\operatorname{Ap}_{\mathrm{G}} \frac{\Delta \mathrm{L}_{\min }}{\Delta \mathrm{P}\left[\left(\frac{\sigma_{\mathrm{V}}}{\mathrm{V}_{\mathrm{G}}}\right)^{2}-\left(\frac{\sigma_{\mathrm{p}}}{\mathrm{p}_{\mathrm{G}}}\right)^{2}\right]^{1 / 2}}
$$

$\mathrm{V}_{\mathrm{MIN}}$ is the minimum in-situ gas volume that is measurable with the given uncertainties in volume and pressure with the given instrument precision. Note that the constraint imposed by Eq. (2.10) must be maintained to keep the denominator of Eq. (2.12) positive.

Figures 2.2 and 2.3 show the detectable void fraction as a function of barometric pressure change for waste configurations representing existing conditions in C-106 and those in AY-102 after transfer, respectively. In each plot an Enraf level gauge with a precision of $1 \mathrm{~mm}$ is assumed

(a) The pressure fluctuation should be only the amount that exceeds twice the yield stress of the material, according to Whitney et al. (1996). But the yield stress in C-106 and AY-102 is expected to be small, $\sim 150 \mathrm{~Pa}$, compared to barometric pressure changes, which typically exceed $1000 \mathrm{~Pa}$. 


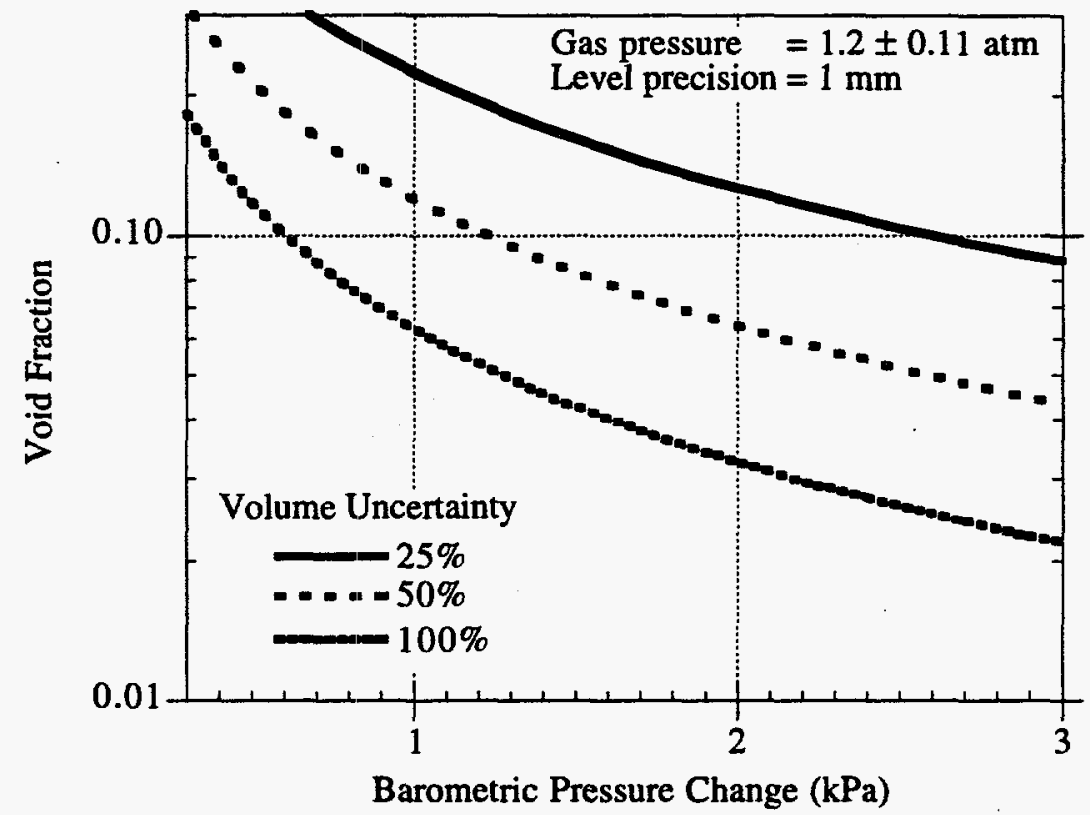

Figure 2.2. Minimum Detectable Void Fraction, C-106 Before Transfer

(analog signal processing). A qualitative indication of stored gas is deemed practical between $100 \%$ and $25 \%$ uncertainty, and good quantitative estimates of gas volume are possible if the volurne uncertainty is less than $25 \%$. The nominal barometric pressure change is about $1 \mathrm{kPa}$, with a maximum of $3 \mathrm{kPa}$ in the winter and a minimum of $0.3 \mathrm{kPa}$ in the summer (Whitney 1995).

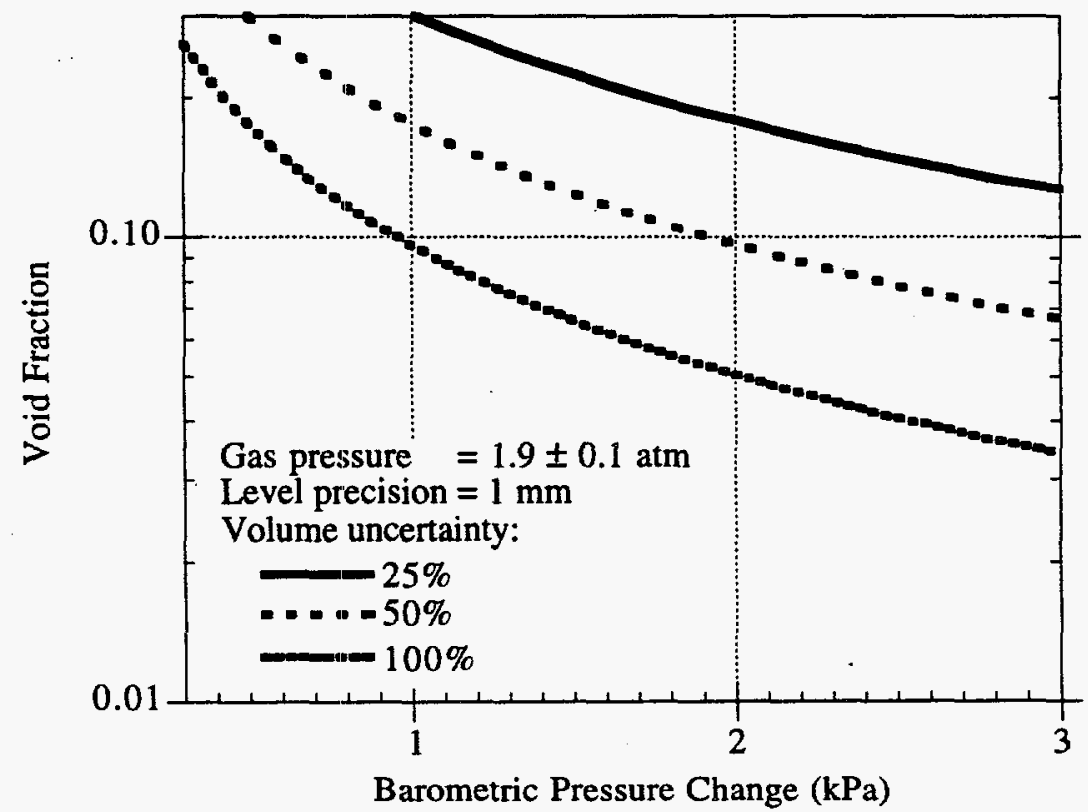

Figure 2.3. Minimum Detectable Void Fraction, AY-102 After Transfer 
The figures show that under nominal barometric pressure swings in the range of $1 \mathrm{kPa}$ the analog Enraf level measurement allows detection of void fractions around 0.10 . Higher pressure swings, on the order of $2 \mathrm{kPa}$, are required to make a quantitative estimate of the gas volume. In Section 3 it is shown that using the digital output directly from the Enraf provides about an order of magnitude higher sensitivity and improves retained gas detection significantly.

The BPE model senses only noncondensible gases. The volume of water vapor in trapped bubbles does not respond to barometric pressure changes because the partial pressure of water vapor is a function only of the local temperature on the time scale of a barometric pressure swing. The water vapor concentration in the bubble grows as temperature increases, acting as a false gas generation. It also causes the water vapor concentration to grow as pressure decreases, slightly amplifying the action of the noncondensible gas.

The water vapor concentration is less than $10 \%$ below waste temperatures of about $60^{\circ} \mathrm{C}$ $\left(140^{\circ} \mathrm{F}\right)$, as shown on Figure 2.4. Above this temperature, water vapor should be accounted for as an important contributor to waste buoyancy and barometric pressure response.

\subsection{Local Void Measurement}

The local void fraction can be measured with the VFI under a riser approximately every $30 \mathrm{~cm}$ (Stewart et al. 1996). The total retained gas volume can be calculated from the average of these local measurements to within about $20-30 \%$, depending on the variability of the waste. The VFI does not measure gas composition. The VFI has the advantage of sampling in a circle of $75 \mathrm{~cm}$ radius around the riser centerline, so its measurements are not compromised by prior core sampling. The VFI is positioned by overhead cranes and does not require truck access.

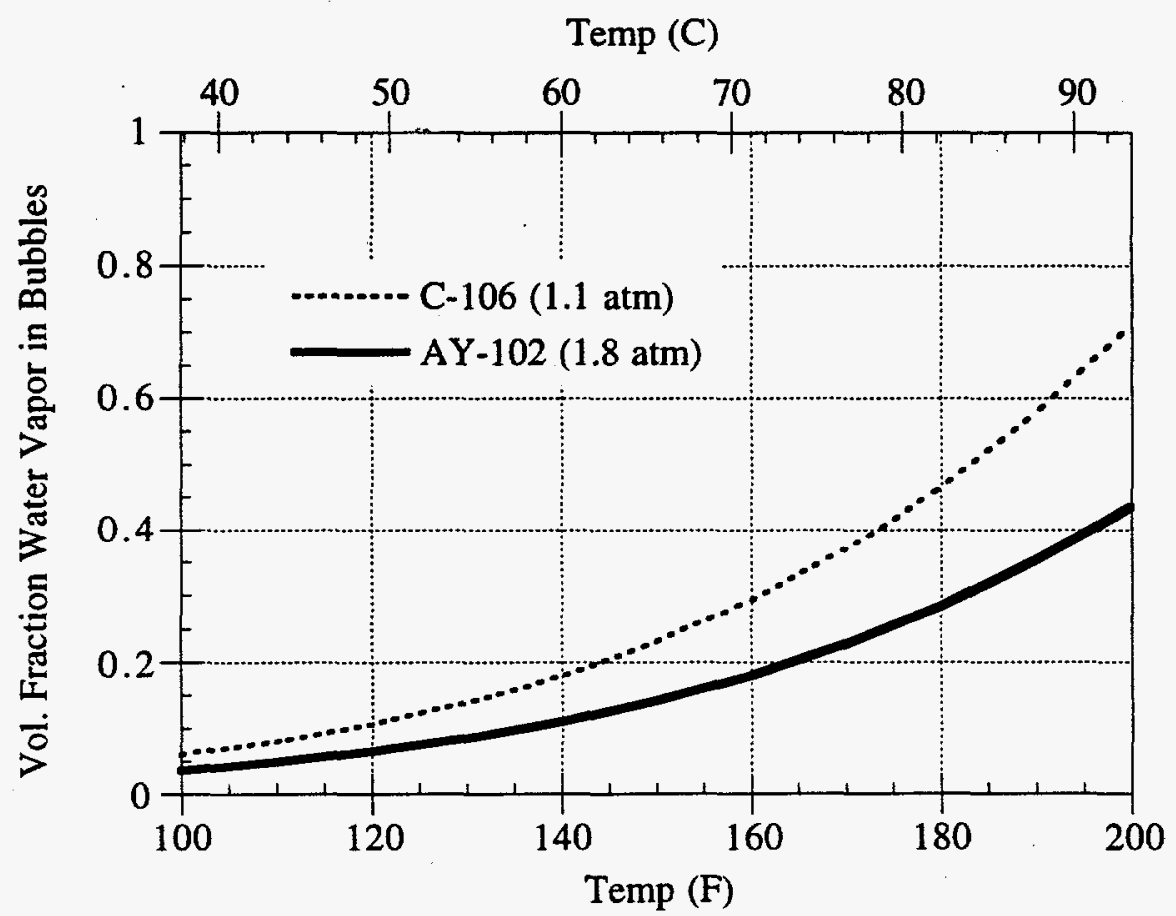

Figure 2.4. Bubble Water Vapor Fraction Versus Waste Temperature 
The void fraction in each 48-cm segment of a push-mode core sample can be obtained with the RGS (Shekarriz et al. 1997). The RGS also provides the composition of the gas. The accuracy of void fraction in individual segments is $\pm 10 \%$ of the nominal void fraction. Because far fewer measurements are made, the uncertainty in the total gas volume calculated from RGS can be much higher than the volume derived from VFI measurements. Also the waste directly under the riser must not have been disturbed by a prior sample, which can severely limit the number of risers available for sampling. The RGS requires access by the push-mode sampling truck. 


\subsection{Measuring Retained Gas in C-106}

The safety assessment for Project W-320 assumes that the gas retention behavior in AY-102 following transfer will be the same as currently exhibited by C-106. Therefore, an estimate of the pretransfer gas inventory in C-106 is required.

The waste surface in C-106 fluctuates with water addition cycles, and long-term trends are difficult to establish. The detectable rise of about $5 \mathrm{~cm}$ since 1979 indicates a possible in-situ gas accumulation of about $800 \mathrm{ft}^{3}$ or a void fraction of $\sim 3 \%$. However, the tank currently appears to be in a steady state in which the gas release rate is equal to the generation rate.

In 1992 the C-106 ventilation flow was shut down, causing the average waste temperature to rise and the waste level to begin increasing. The partial pressure of water vapor in the retained gas bubbles is a function only of the local temperature, and the volume change with temperature depends on the fraction of noncondensible gas present. Therefore, a noncondensible gas volume can be estimated if the surface level rise rate (corrected for evaporation) and the rate of rise of the volume average temperature are known. This calculation for the 1992 outage yielded gas volumes ranging from $1000-3000 \mathrm{ft}^{3}$. The wide range results from uncertainties in evaporation and average temperature.

\subsection{Barometric Pressure Response}

The barometric pressure response in C-106 has been difficult to assess because of the scatter in the Enraf level gauge analog signal output compared with the relatively small changes in level. The precision available with the current system is $0.1 \%$ of the total level span of 50 inches. This reduces to 0.05 in., or $1.3 \mathrm{~mm}$. The sensitivity of raw digital signal is more than an order of magnitude higher at $0.1 \mathrm{~mm}$. ${ }^{\text {(a) }}$ It appears that the actual precision current measurement is $-0.5 \mathrm{~mm}$, which is at least a factor of 2 better than expected from the analog signal. This is shown by the level history for January 8 and 9, 1997, in Figure 3.1.

Based on the discussion in Section 2, the current Enraf data are useful for estimating gas volume in C-106 only for periods in which large barometric pressure swings occur. One such two-week period occurred in November 1995. The average $\mathrm{dL} / \mathrm{dP}$ computed from the detrended (to remove the effect of evaporation) data was $-0.12 \pm .075 \mathrm{~cm} / \mathrm{kPa}$. Assuming a gas pressure of $110 \mathrm{kPa}$, the in-situ gas volume is $2000 \pm 1200 \mathrm{ft}^{3}$ and the void fraction is $7.5 \pm 5 \%$. This level of uncertainty is consistent with the gas volume uncertainty predicted by Eq. (2.10) and the void fractions plotted in Figure 2.2.

The current digital-to-analog conversion will be bypassed, and raw digital data will be recorded directly to provide a clean correlation of surface level and barometric pressure. After this modification, if the expected $0.1-\mathrm{mm}$ precision can be achieved, gas volume fractions down to a few percent should be measurable with a relative uncertainty better than $50 \%$ under only nominal barometric pressure fluctuations, as shown graphically in Figure 3.2.

(a) Personal communication with JH Bussel, Numatec Hanford Company, July 10, 1997. 


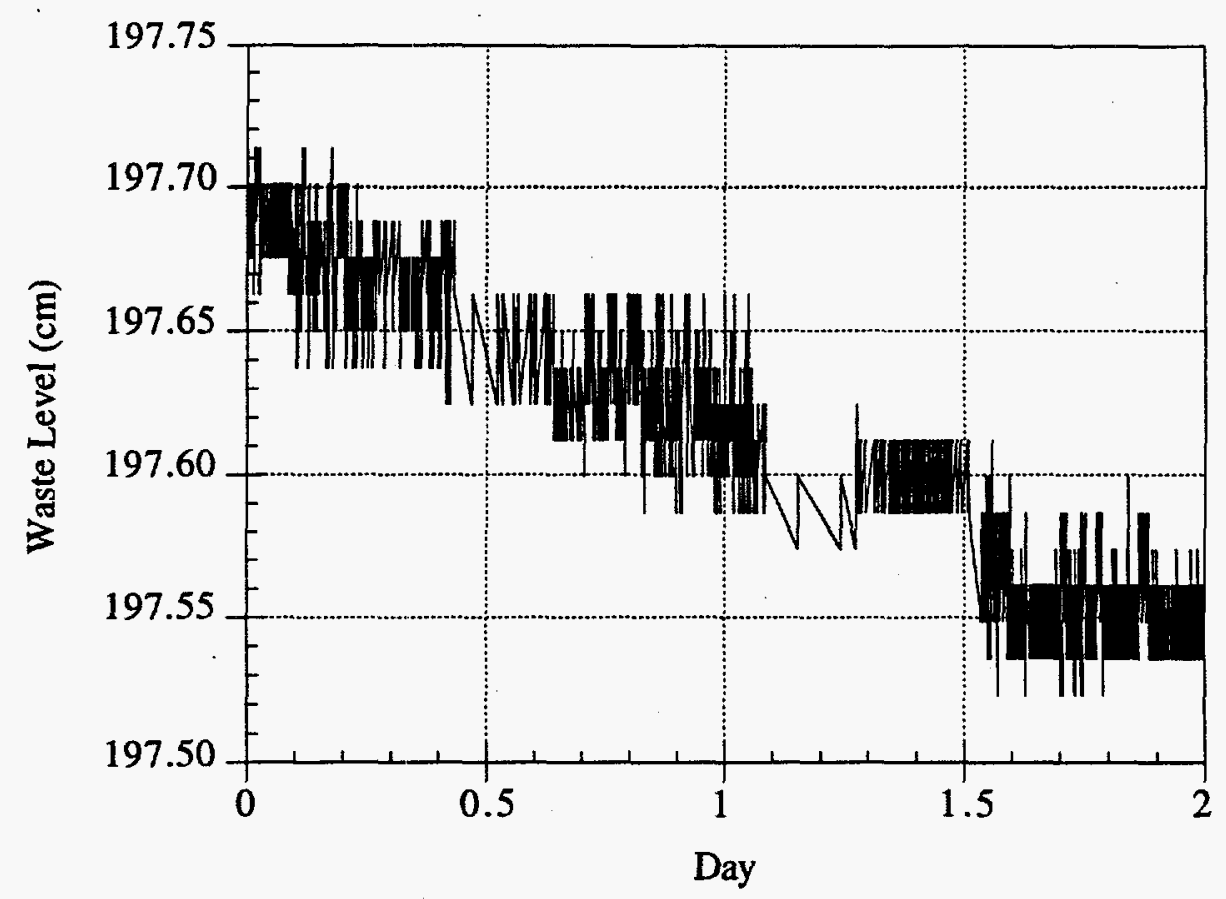

Figure 3.1. Enraf Surface Level History for C-106: January 8 and 9, 1997

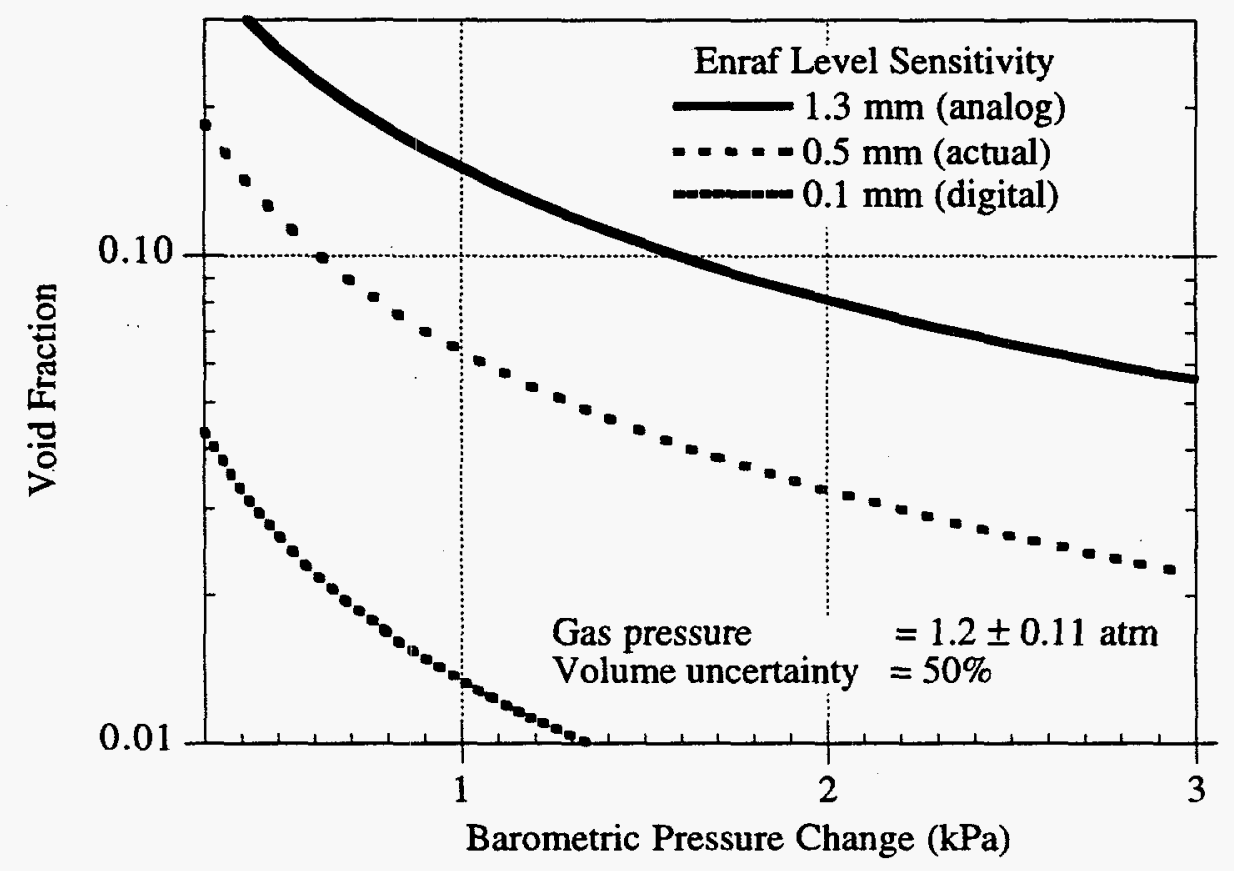

Figure 3.2. Void Fraction Detectable via BPE in C-106 for Several Level Sensitivities 
Based on this and the rough estimates above, we estimate that $\mathrm{C}-106$ contains at least $1000 \mathrm{ft}^{3}$ of retained gas, a void fraction of $\sim 4 \%$ but less than $4000 \mathrm{ft}^{3}$, and a void fraction of $15 \%$. Video recorded while positioning the transfer pump showed that a significant swarm of bubbles was released when the upper layers of waste were disturbed. This is consistent with void fractions in this range.

\subsection{Gas Released During Sluicing}

It is possible to estimate the gas volume in C-106 after the fact by measuring the gas volume released when the waste is disrupted by sluicing. The standard gas release volume is simply the product of the headspace exhaust flow rate and the headspace hydrogen concentration divided by the estimated hydrogen fraction of the waste gas:

$$
\hat{\mathrm{V}}_{\mathrm{REL}}=\dot{\mathrm{Q}}_{\mathrm{VENT}} \Delta \mathrm{t} \frac{\Delta\left[\mathrm{H}_{2}\right]_{\text {Dome }}}{\left[\mathrm{H}_{2}\right]_{\text {Waste }}}
$$

The uncertainty in the release volume via Eq. (3.1) is estimated to be about $30 \%$, dominated by a $20-25 \%$ uncertainty in the vent flow rate.

The mass flow rate and solids fraction of the slurry transferred to AY-102 will be measured by a Coriolis flowmeter (see Section 5) as it moves through the line. The volume of C-106 sludge that is actually disturbed to create this volume of solids is computed by

$$
\mathrm{V}_{\mathrm{SL}}=\mathrm{F}_{\mathrm{D}} \Delta \mathrm{t} \frac{\dot{\mathrm{m}}_{\mathrm{FM}}\left(\chi_{\text {solids }}\right)_{\mathrm{FM}}}{\rho_{\mathrm{SL}}\left(\chi_{\text {solids }}\right)_{\mathrm{SL}}}
$$

where $\dot{\mathrm{m}}_{\mathrm{FM}}$ is the mass flow rate, $\left(\chi_{\text {solids }}\right)_{\mathrm{FM}}$ is the solids weight fraction measured by the flowmeter, and $\left(\chi_{\text {solids }}\right)_{S L}$ is the estimated solids weight fraction in the undisturbed C-106 sludge. The density of the C-106 sludge is $\rho_{S L}$, and $F_{D}$ is a factor accounting for the excess sludge volume disturbed, and therefore degassed, beyond what is actually transferred.

The void fraction is computed from Eq. (3.1) and (3.2) by the standard definition

$$
\alpha=\frac{\mathrm{V}_{\mathrm{REL}}}{\mathrm{V}_{\mathrm{REL}}+\mathrm{V}_{\mathrm{SL}}}
$$

where the in-situ volume, $\mathrm{V}_{\mathrm{REL}}$, is determined from the standard volume in Eq. (3.1) and effective pressure ratio via Eq. (2.5).

The $F_{D}$ factor is the source of most of the uncertainty in the sludge volume calculation. In the initial transfer of the equivalent of $2 \mathrm{ft}$ of C-106 sludge, it is likely that $4 \mathrm{ft}$ will actually be disturbed (see Section 5.1). If $F_{\mathrm{D}}$ ranges from 1-3 with a nominal value of 2 , its uncertainty might be $-50 \%$. The uncertainty in the sludge volume without considering $\mathrm{F}_{\mathrm{D}}$ is just over $30 \%$, dominated by the $30 \%$ error estimated for the solids weight fraction in the slurry. The resulting error in the void fraction could be as high as $100 \%$. However, at some point when the transfer is nearly complete the $\mathrm{F}_{\mathrm{D}}$ factor approaches unity, and the uncertainty in the calculated void fraction reduces to $-40 \%$. 
Therefore, the gas released during sluicing can be taken as a fairly accurate indicator of the average void fraction in C-106 after most of the waste is transferred. However, it should not be used as a quantitative calculation in the initial stages of the operation.

\subsection{VFI/RGS Void Fraction Measurements}

The gas volume stored in C-106 could theoretically be determined most accurately from local measurements with the VFI or RGS. There are no accessible risers available for the RGS since they have been contaminated by earlier core samples or by intrusive equipment that would need to be removed.

The VFI samples outside the riser centerline, so previous core samples do not compromise a riser. The VFI would probably be able to penetrate the C-106 sludge without problems. However, the only risers available to the VFI are near the tank wall and samples taken there would not be representative of the whole tank. Also, the radiation dose to the workers limits riser selection to those outside the pits. So it simply is not practical to attempt to measure void fraction in C-106 with the VFI. 


\title{
4.0 Monitoring Gas Retention in AY-102 After Transfer
}

\author{
AY-102 shows no barometric pressure response nor is there any other indication of trapped \\ gas. However, since the tank contains only about $30 \mathrm{~cm}$ of nonconvective material that could \\ retain gas, even a $15 \%$ void fraction would represent a gas volume well below the minimum \\ detection limit.
}

Some gas retention is expected in AY-102 following transfer of waste from C-106. The steady-state void fraction is expected to be $5-10 \%$, the same as is currently estimated to be in C-106. This will not approach neutral buoyancy, and no large episodic gas releases are expected. The neutral buoyancy void fraction in AY-102 following transfer could be as high as $30 \%$, assuming the lighter carbonates and aluminates dissolve. It is extremely unlikely that the relatively shallow nonconvective layer in AY-102 could attain this high a void fraction.

On the other hand, if less solids dissolve and the settled layer settles more loosely than the original sludge in C-106 (is "fluffed"), the neutral buoyancy void fraction could be as low as $20 \%$. Therefore it may be possible, though improbable, for the nonconvective layer to reach such a high void fraction that large gas releases via the buoyant displacement mechanism could not be ruled out (Meyer et al. 1997).

Buoyant displacements can be prevented by removing the supernatant liquid. This prevents the release of buoyant energy that fluidizes the sludge and releases the gas. Removal of supernatant liquid also has the benefit of reducing the hydrostatic pressure and increasing the headspace so that the consequences of any releases that might occur would be much less severe. Therefore, the main concern for monitoring gas retention in AY-102 is the decision whether or when to start removing supernatant liquid.

There are several methods available to measure gas retention in AY-102. All of these methods have significant uncertainties, but together they form a preponderance of evidence that describes the gas retention behavior of the tank. The available methods are the BPE model, the SLE model, growth of the nonconvective layer, comparing gas generation with release rates, and direct void fraction measurement with the VFI or RGS. Each method will be discussed in turn.

\subsection{Barometric Pressure Response}

The BPE method has a somewhat higher uncertainty in AY-102 after transfer than it did in $\mathrm{C}-106$ because of the increased waste depth and resulting higher hydrostatic pressure. This is illustrated in Figure 4.1. An Enraf level gauge will be installed in the tank prior to transfer. Using the sensitivity ascribed to an analog signal, void fractions on the order of $10 \%$ can be detected during the larger barometric pressure swings. If the digital output is recorded, with its higher sensitivity the BPE model could measure voids of about $5 \%$ with a relative uncertainty better than $50 \%$ under nominal barometric pressure swings.

The water vapor fraction, which the BPE model does not sense, is not expected to be a significant contributor in AY-102 if the cooling systems perform as anticipated and the waste temperature remains low (see Figure 2.5). If the temperature rises to the point that the water vapor fraction becomes significant, the large number of temperature measurements available in the tank will make it possible to compensate accurately. 


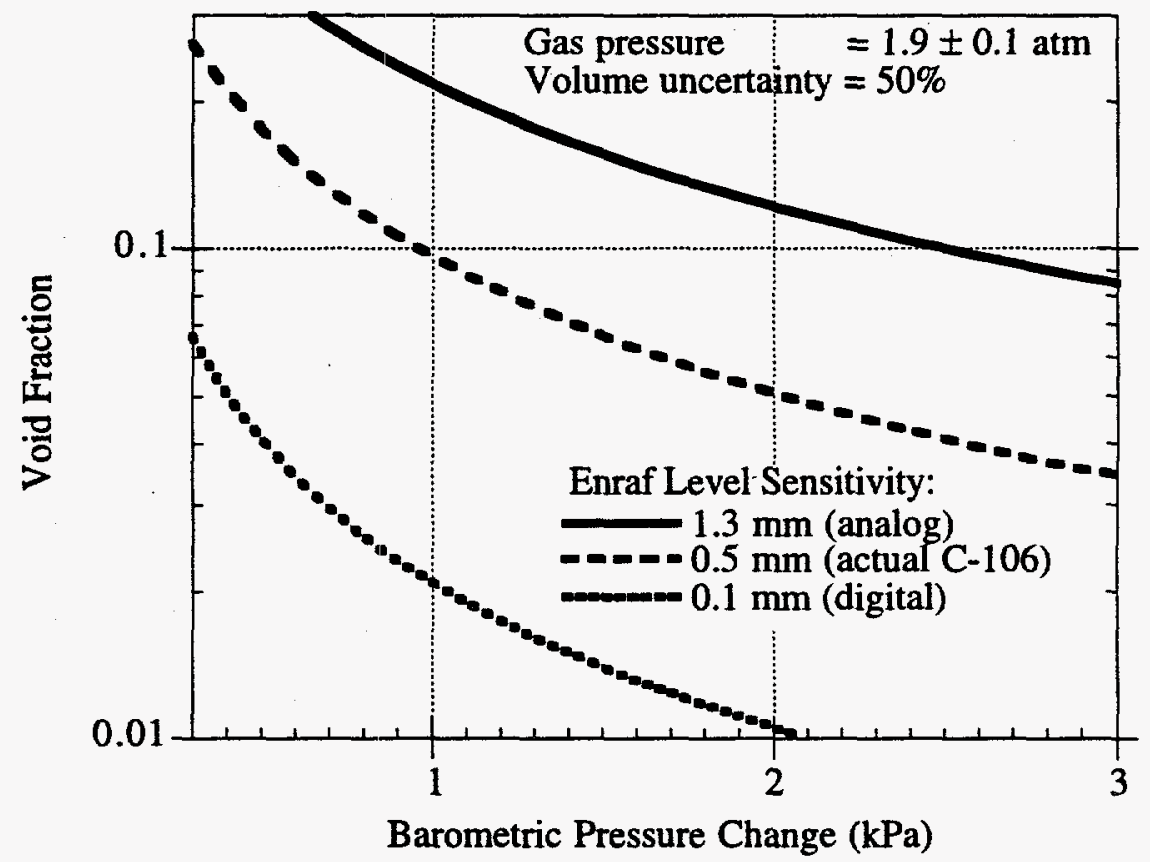

Figure 4.1. Void Fraction Detectable via BPE in AY-102 for Several Level Sensitivities

\subsection{Surface Level Rise}

The method of estimating the retained gas volume via waste surface level rise was discussed in Section 2. The fractional error in retained gas volume depends on the fractional error in the evaporation rate and the ratio of evaporation rate to retention rate according to Eq. (2.4). Assuming that half the gas generated is retained and is held at an average pressure of $1.8 \mathrm{~atm}$ in AY-102 and that the evaporation rate is known to within $\pm 30 \%$, Eq. (2.4) predicts the fractional uncertainty in the retained gas volume, as shown in Figure 4.2.

The gas generation rate in AY-102 following transfer could range from 30-60 scf/day. Evaporation in AY-102 could range from its current rate of $\sim 0.7 \mathrm{in} . / \mathrm{mo}$ up to $\sim 3 \mathrm{in} . / \mathrm{mo}$ when it eventually reaches a thermal steady state. This range is indicated by the shaded area in Figure 4.1, which corresponds to a 30-60\% uncertainty in gas retention as determined by the SLE method. The uncertainty is inversely proportional to the fraction of generated gas that is retained.

However, the ventilation system in AY-102 will be modified to increase the annulus cooling flow significantly and to add a recirculating chiller system to the headspace. The latter improvement will greatly reduce the exhaust flow and the headspace humidity. Thus the evaporation rate is expected to be significantly lower when transfers begin. Assuming that the chiller maintains the headspace at a temperature of $80^{\circ} \mathrm{F}$ and at $70 \%$ relative humidity, with an exhaust flow of $100 \mathrm{cfm}$ the evaporation rate will be cut to about $0.25 \mathrm{in} . / \mathrm{mo}$. If this is true, the retained gas volume estimated by the SLE model may be accurate to within $\pm 10 \%$. 


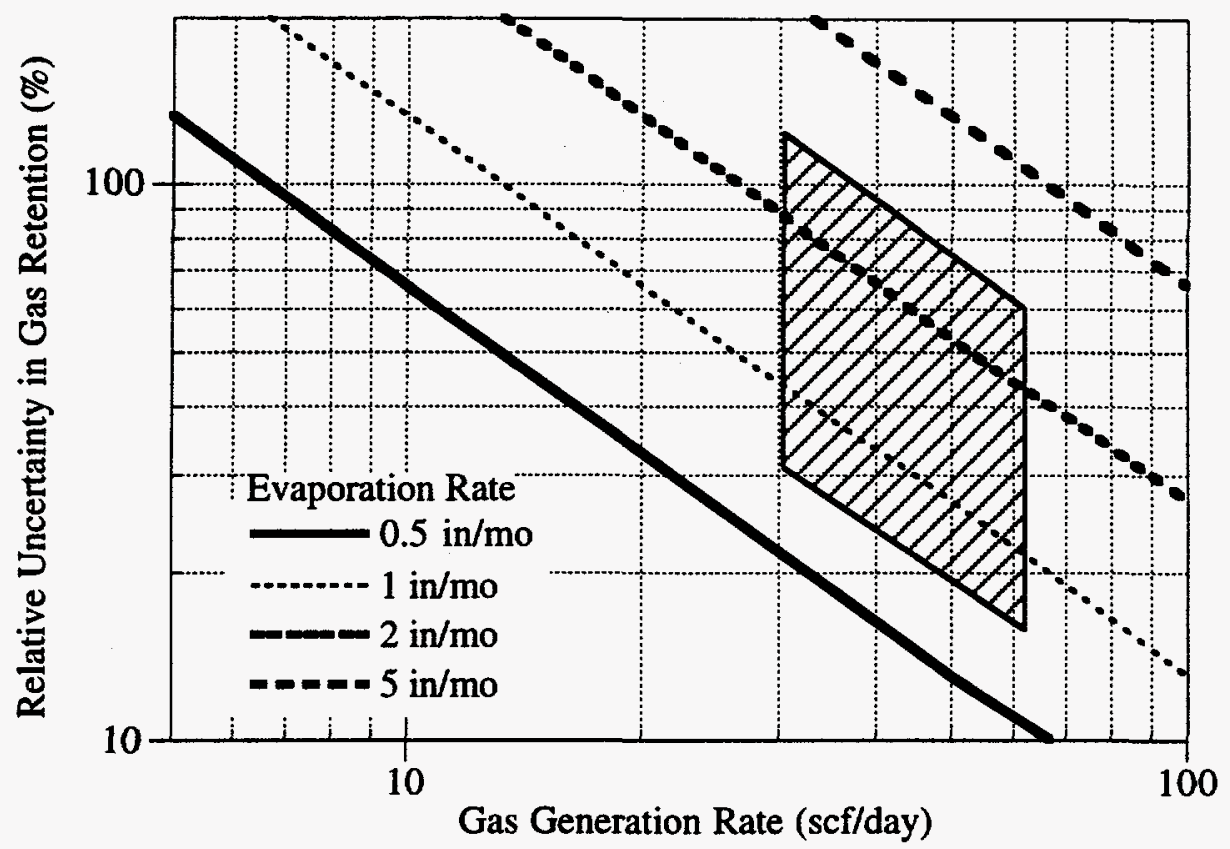

Figure 4.2. Uncertainty in Gas Retention via the Surface Level Effect Model

Regardless of the evaporation rate, a significant measured surface level rise is probably an accurate indication of significant gas retention. But, the smaller the level rise, the larger the uncertainty in the retained gas volume that can be computed from it.

\subsection{Conservation of Gas Generation in C-106 and AY-102}

The gas generation rates of both tanks will be well established prior to transfer by using the recently installed gas monitors with the measured ventilation rates. If AY-102 does not retain gas, the sum of the gas generation rates before transfer should be approximately equal to the sum of release rates measured after transfer. If the post-transfer sum is less, one can deduce that AY-102 is retaining gas at a rate about equal to the difference. This is expressed by

$$
\dot{\mathrm{V}}_{\mathrm{RET}}=\left(\dot{\mathrm{V}}_{\mathrm{AY} 102}\right)_{\text {Before }}+\left(\dot{\mathrm{V}}_{\mathrm{C} 106}\right)_{\text {Before }}-\left(\dot{\mathrm{V}}_{\mathrm{Cl} 106}\right)_{\text {After }}-\left(\dot{\mathrm{V}}_{\mathrm{AY} 102}\right)_{\text {After }}
$$

There is significant uncertainty in this method. Some increases in gas generation rate are expected in AY-102 because the waste will be much more dilute and more water will be available for radiolysis. Therefore, the success of this method depends on how well this change can be determined. If there is no change in the specific gas generation rate, assuming each of the generation rates in Eq. (4.1) is known to within $\pm 30 \%$, the retention rate estimated for AY-102 will be accurate only to within $\pm 50 \%$. Therefore, this method should be considered only a qualitative indicator of gas retention in AY-102 at best. 


\subsection{VFI/RGS Void Fraction Measurements in AY-102}

The gas volume that accumulates in AY-102 after transfer can be determined relatively accurately from local measurements with the VFI or RGS. There are ample risers available, and the waste will be relatively uniform, so a local measurement would be representative of the entire tank with a high probability.

However, it is not useful to attempt to operate either of these devices during the initial stages of transfer. First, not enough gas will have accumulated to be measured or to be significant in predicting future retention. Second, the RGS sample is 19 inches in length, so only one or two samples would be available following the first $2 \mathrm{ft}$ of transfer. The VFI similarly requires at least $2 \mathrm{ft}$ of waste for an effective measurement ( $1 \mathrm{ft}$ above and below the sample location). Therefore, neither the VFI nor the RGS should be used until after complete transfer and then only after there are other indications of significant gas retention. 


\subsection{Monitoring Slurry Transport}

Transferring sludge-type waste from one tank to another requires several basic steps. First the sludge is slurried and its physical properties brought to within the acceptable ranges for safe transport. It is then introduced into the transfer pipeline through a slurry pump that is capable of imparting sufficient pressure to the slurry medium to allow it to travel the necessary distance while still in a well-mixed state. The slurry should be discharged close to the top of the existing nonconvective layer in the receiver tank to speed settling of the transported solids. The discharged slurry should be spread over as much as possible of the tank cross-sectional area to avoid forming separate sludge mounds and supernatant puddles. This occurrence may cause the returning supernatant liquid to bring solids back to the original tank, an undesirable and wasteful result.

This section presents a short discussion on the mechanics of the sluicing operation as dictated by the planned sluicing configuration. The slurry instrumentation requirements have considerable impact on detecting flammable gas and predicting the steady-state temperature in AY-102. Two classes of process control instrumentation will be discussed; the first is designed to measure and monitor parameters related to the amount of slurry being transferred, and the second measures and monitors the heat load being transferred through the slurry pipeline. Both are designed to ensure controlled, reliable, and safe operation of the transfer process.

\subsection{Sluicing Operations}

Many of the process control parameters used in Project W-320 rely on the assumption that a 2-ft-thick layer of sludge will be removed from Tank C-106 and transported in slurry form to Tank AY-102. Sludge mobilization will be accomplished by sluicing, and a slurry pump will retrieve the mobilized sludge and deliver it to the transport pipeline. Slurry concentrations by volume that vary from 10 to $30 \%$ solids are expected during the retrieval and transfer operations.

A single sluicing nozzle will be located approximately $5 \mathrm{ft}$ from the surface of the internal wall of Tank C-106, and a slurry pump will be located at a diametrically opposed position, also $5 \mathrm{ft}$ from the tank wall. This means that the sluicing jet is always at a horizontal distance of $65 \mathrm{ft}$ from the slurry pump. While the slurry pump can be raised or lowered vertically, the sluicing jet nozzle elevation relative to the waste is fixed. It is assumed that the nozzle is mounted on a hinged arm with the hinge permanently about $9 \mathrm{ft}$ above the surface of the sludge. The nozzle itself will be capable of rotating about that hinge in a vertical plane and can be set at a prescribed negative angle from the horizontal. It is anticipated that the nozzle axis will always be positioned at angles $\alpha \leq 0$ relative to the horizontal. The vertical riser that supports the nozzle/hinged arm assembly can also rotate about its own axis to allow the nozzle to sweep over a conical surface when $\alpha<0$, to cover all parts of the cylindrical waste volume.

Figure 5.1 depicts the sluicing jet, the slurry pump intake, and the waste cylinder of initial depth $h$. The waste volume mobilized by the sluicing jet, placed at height $\mathrm{H}$ above the tank bottom at the indicated sluicing nozzle orientation, is bounded by the waste cylinder to a depth $\delta$ and by the conical surface generated by the nozzle sweeping lateral movement at the angle $\alpha$. This volume is equivalent to the volume of a cylindrical slice of sludge with a uniform thickness $\eta$. 


\section{Sluicing Jet Nozzle}
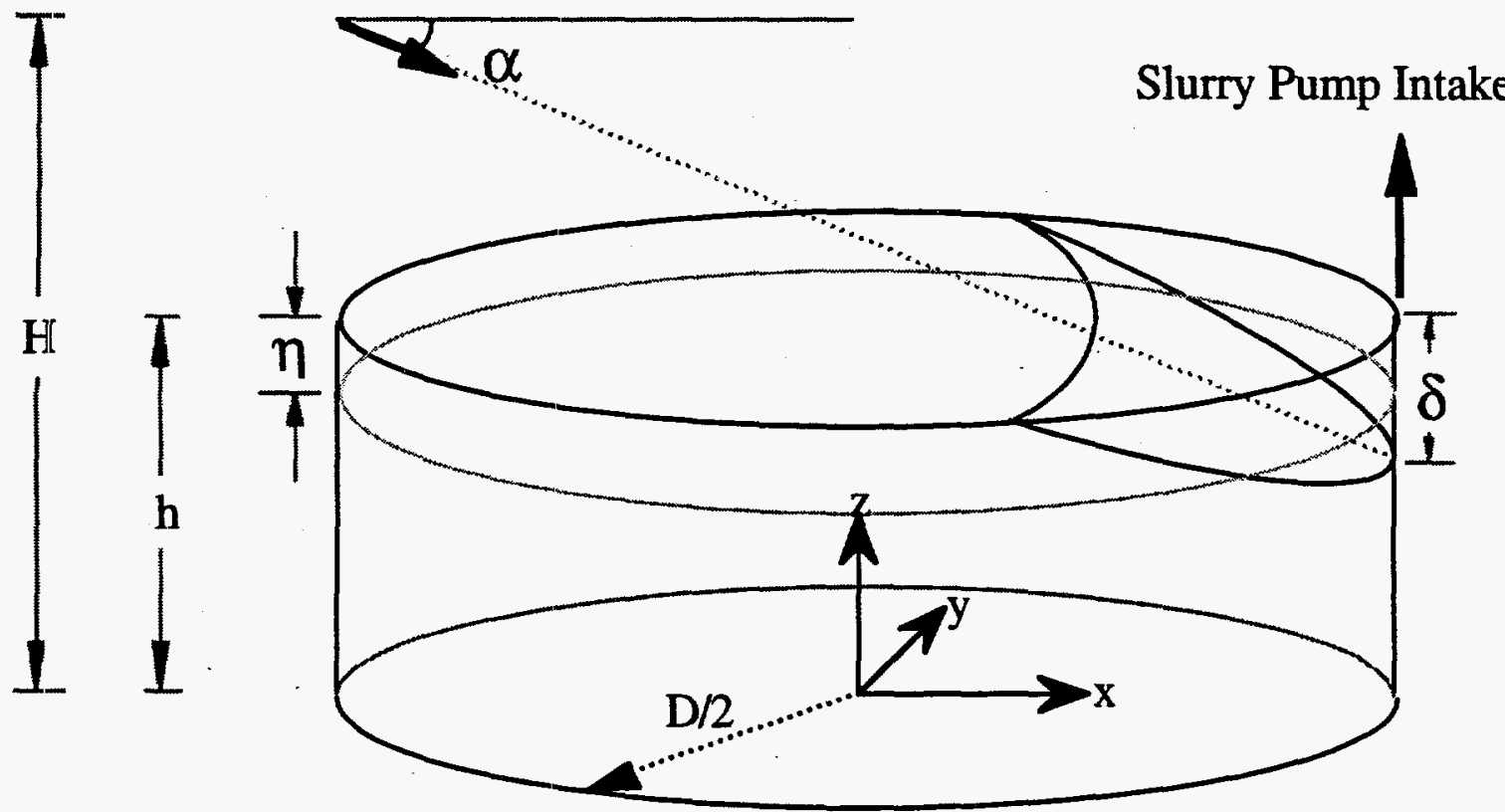

Figure 5.1. Schematic of Sludge Cylinder with Sluicing Jet and Slurry Pump Locations (no overlayer of supernatant liquid shown)

Theoretical development has been done to relate the two volumes and give a comprehensive description of the generated surfaces for various sluicing jet heights and locations relative to the edge of the waste cylinder. ${ }^{(a)}$

To illustrate, Figure 5.2 shows the situation in which the sluicing jet is confined to mobilizing waste sludge within the upper 2 - $\mathrm{ft}$ layer. In this case $\alpha=9.6^{\circ}$ and $\delta=2 \mathrm{ft}$ with a removed sludge volume of 3455 gallons. To remove a sludge volume of 50,000 gallons, which represents a uniform cylindrical slice of thickness $\eta=2 \mathrm{ft}$, the sluicing nozzle must remove the sludge all the way to the bottom of the waste cylinder and advance back a considerable distance, as shown in Figure 5.3. In this case, $\alpha=15^{\circ}$ and $\delta=h=6 \mathrm{ft}$ with a removed sludge volume of 50,000 gallons. Figure 5.4 shows the solution to the developed model for $h=6 \mathrm{ft}$ and $\mathrm{H}=15 \mathrm{ft}$, which represents the nozzle location in Tank C-106.

(a) Erian FF, LA Mahoney, and G Terrones. 1997. Analysis of Single Jet Sluicing of Tank Waste (draft). Pacific Northwest National Laboratory, Richland, Washington. 


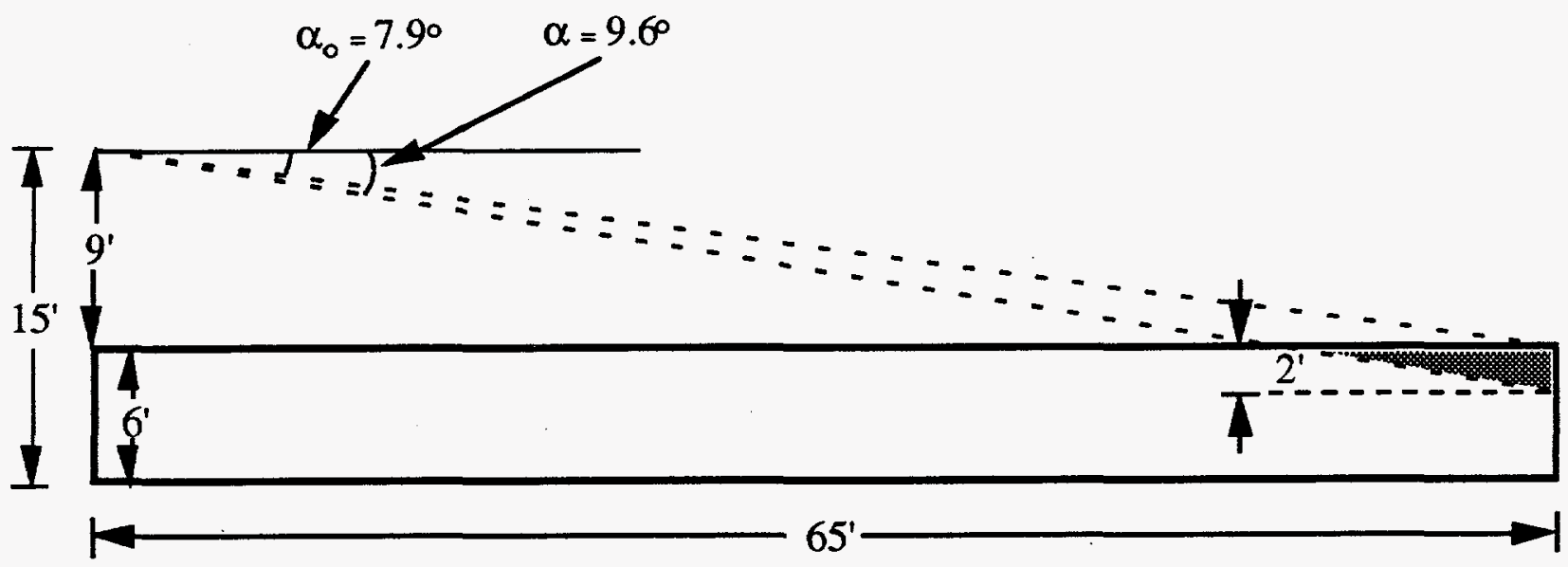

Figure 5.2. Sludge Removed when 2-ft Depth Is Reached at Pump End

It is not realistic to expect that a uniform sludge layer of thickness $\eta$, as shown in Figure 5.1, will be removed from the waste cylinder, considering the geometry and locations of the sluicing jet and the retrieval pump. An inclined surface (a grade) directed toward the retrieval pump must be generated and maintained by the sluicing jet to let gravity currents move the mobilized slurry from very near the sluicing nozzle to near the slurry pump. Agitation and currents due to pump suction will have some effect but are more likely to mobilize sludge immediately below or near the pump intake. It is not plausible for the pump suction to generate currents strong enough to move the mobilized sludge from the opposite side of the tank without the grade (gravity).

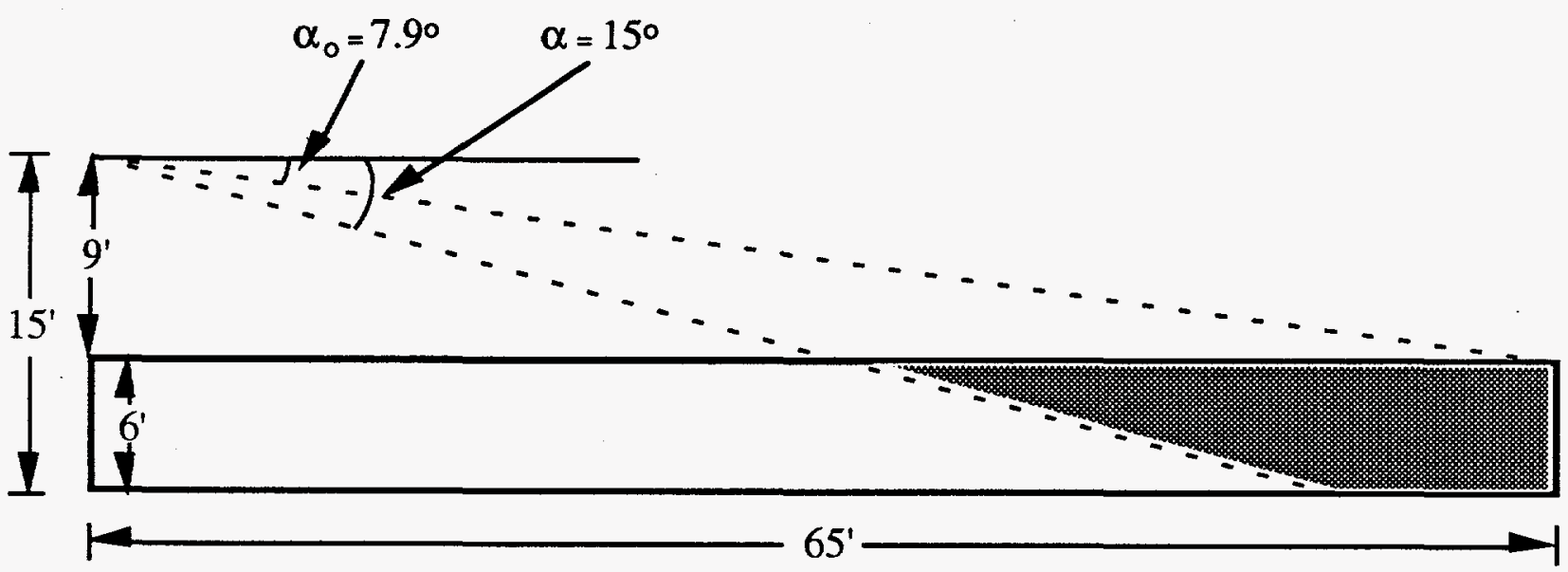

Figure 5.3. Sludge Removed Equivalent to $2-\mathrm{ft}$ Uniform Slice, $50,000 \mathrm{ft}^{3}$ 


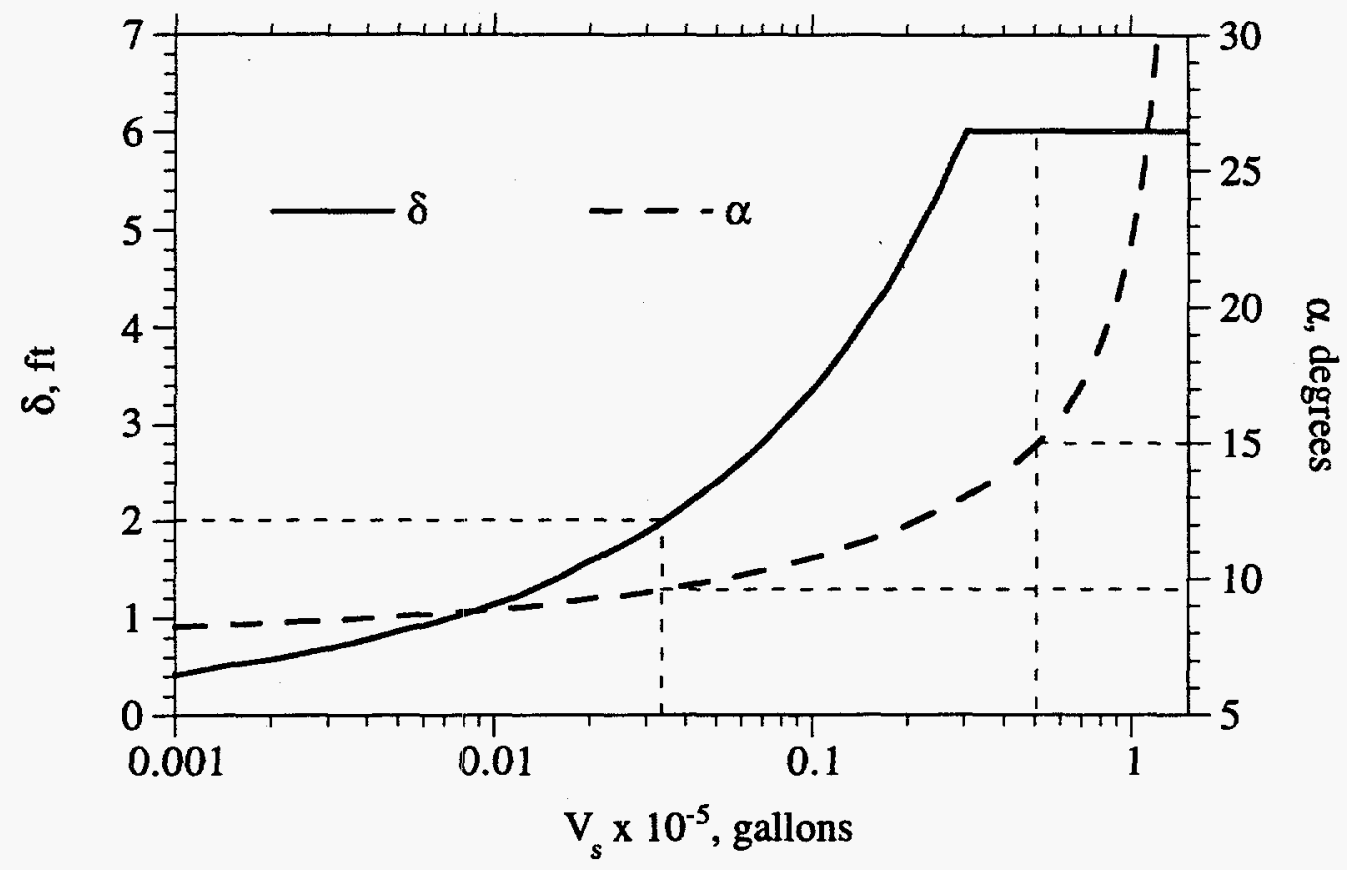

Figure 5.4. Calculated Sludge Volumes, $\mathrm{V}_{\mathrm{s}}$, Removed by Sluicing

Creating the required grade will disturb the sludge to a greater depth than was planned for. To remove the top $2 \mathrm{ft}$ it may be necessary to disturb $4 \mathrm{ft}$ or more near the pump. A higher than expected heat load may be transferred in the first 2-ft stage because the concentration of heatgenerating isotopes increases with depth. Many of the heat transfer calculations may be influenced by the practical aspects of solids removal via the installed sluicing/retrieval geometry. Also, it will not be practical to determine the original gas content of $\mathrm{C}-106$ from the volume of gas released during sluicing until most of the waste has been removed. Section 3 contains a complete discussion of this subject.

Sludge mobilization is made even more difficult by the need to retain a 2-ft layer of supernatant liquid above the sludge surface to maintain hydrostatic pressure. The supernatant liquid will reduce the effectiveness of the sluicing jet and the ability to maneuver and "massage" the sludge surface, especially at large distances from the nozzle exit. This may cause the retrieved slurry to have considerably lower solids concentration. A potential improvement to the sluicing process would be to allow the sluicing nozzle support riser to be lowered in a controlled manner from its planned fixed position so the sludge can be "massaged" by the water jet to achieve the shallowest possible grade between the nozzle and the pump.

\subsection{Operational Process Control Instrumentation}

Process control instrumentation for slurry transport is built into the system at the pump pit of each tank. In C-106 it is possible to monitor the electric current through the slurry pump's drive motor as a measure of the pressure drop across the transfer line. Small current fluctuations are expected due to the anticipated variations of the average slurry density during the sluicing and retrieval operations. The nature of the sluicing process and the differing solubilities of the various 
solid constituents of the sludge make it impossible for the solids concentration and the specific gravity of the carrier liquid to remain fixed throughout the transfer process. A Red Valve ${ }^{\circledR}$ pressure transducer is located in the same pump pit to measure the pressure at the transfer line inlet. The value of this gauge's reading would correspond to the electric current consumption of the pump's drive motor, assuming the pressure at pipeline discharge is nearly atmospheric.

In the pump pit of Tank AY-102 a new instrument is being installed that will measure the mass flow rate of the slurry and its average density. This device, known commercially as a Micromotion mass flowmeter, will be installed at the end of the transfer line. The slurry will be homogenized (e.g., with a static mixer) before it enters into this double-tube instrument to ensure that measurements are accurate within the manufacturer's specifications.

\subsubsection{The Micromotion Instrument as a Process Control Device}

The Micromotion flowmeter measures fluid mass in motion and the average density of the fluid within its sensor's flow tubes. In this case this "fluid" is a slurry, i.e., a liquid-solid mixture. The flow tubes receive mechanical excitation that causes them to oscillate in a free vibration mode at their natural frequency (similar to a tuning fork). The amplitude of the oscillations is very small relative to the length of the flow tubes so the motion can be modeled accurately as a linear springmass system. The average density of the slurry within the flow tubes can be shown to be inversely proportional to the square of their natural frequency.

The flow of the slurry into and out of the vibrating flow tubes generates a tortional or twisting cyclic motion (due to the Coriolis effect) whose amplitude is directly proportional to the mass flow rate. A major benefit of this principle is that changes in the slurry's physical properties, such as temperature, pressure, density of carrier liquid, viscosity, and conductivity, have no effect on the accuracy of the measurement of the mass of the flowing slurry by this device.

The main reason for installing the Micromotion mass flowmeter on the transfer line is not to obtain the slurry's mass flow rate and its average density but rather to obtain process control information on the amount of solids being transferred from C-106 to AY-102. Two important process control parameters can be derived from the output data of the flowmeter when combined appropriately with the densities of the slurry's carrier liquid and solids. The first process control parameter considered here is the sluicing time, $\tau, \mathrm{hr}$, which is defined as the time required to transfer a specified amount of solids, $\mathrm{V}_{\mathrm{s}}, \mathrm{ft}^{3}$

where

$$
\tau=\frac{\mathrm{V}_{\mathrm{s}}}{\left(\frac{\dot{\mathrm{m}}}{\bar{\rho}}\right)_{\mathrm{C}_{\mathrm{v}}}}, \mathrm{hr}
$$

$\tau=$ sluicing time, hr

$\mathrm{V}_{\mathrm{s}}=$ solids volume targeted for removal, $\mathrm{ft}^{3}$

$\dot{\mathrm{m}}=$ measured slurry mass flow rate, $\mathrm{ft}^{3} / \mathrm{hr}$

$\mathrm{C}_{\mathrm{v}}=$ solids concentration by volume

$$
=\left(\frac{\bar{\rho}-\rho_{\ell}}{\rho_{s}-\bar{\rho}}\right)
$$


Knowledge of $\rho_{\ell}$ and $\rho_{s}$ allows the calculation of $C_{v}$ using the measured value of $\bar{\rho}$. The accuracy of the values we have for the supernatant liquid density, $\rho_{\ell}$, and the material density of the solid particles in the sludge, $\rho_{s}$, control the accuracy with which we are able to determine $C_{v}$. The uncertainty in $\rho_{\ell}$ is relatively high because we have insufficient information on the solubilities of the different solids constituents in the sludge.

Sluicing operations cannot maintain a steady value for $\mathrm{C}_{\mathrm{v}}$. However, changes would be relatively slow, so the Micromotion flowmeter average slurry density and mass flow rate outputs can easily follow these changes. Given a target amount of solids, $\mathrm{V}_{\mathrm{s}} \mathrm{ft}^{3}$, to be removed from C-106, the required time can be estimated from Eq. 5.1 .

The uncertainty in the sluicing time has been estimated. ${ }^{\text {(a) }}$ The information shows that even with accurate readings from the Micromotion flowmeter, large uncertainties will exist in the estimates of sluicing time due to the high uncertainties in the carrier liquid density, $\rho_{\ell}$. The calculations were based on the following conditions:

$\begin{array}{ll}\text { Volume of solids to be transported, } \mathrm{V}_{\mathrm{s}} & =2272.57 \mathrm{ft}^{3} \\ \text { Mass flow rate of slurry, } \dot{\mathrm{m}} & =\text { depends on } \mathrm{C}_{\mathrm{v}}, \mathrm{lb}_{\mathrm{m}} / \mathrm{hr} \\ \text { Density of the carrier liquid, } \rho_{\ell} & =67.77 \mathrm{lb}_{\mathrm{m}} / \mathrm{ft}^{3} \\ \text { Density of the solid particles, } \rho_{\mathrm{s}} & =124.8 \mathrm{lb}_{\mathrm{m}} / \mathrm{ft}^{3}\end{array}$

The uncertainties in the above quantities were given by

$\Delta \mathrm{V}_{\mathrm{s}}= \pm 0\left(\mathrm{~V}_{\mathrm{s}}\right.$ is the target volume to be removed $)$

$\Delta \rho_{\ell}= \pm 1.355$

$\Delta \rho_{\mathrm{s}} \quad= \pm 12.48$

$\Delta \bar{\rho} \quad= \pm 0.0312$

$\Delta \dot{\mathrm{m}}= \pm 0.0757$

Figure 5.5 shows the contributions of the uncertainties in the different parameters that contribute to the estimated sluicing time, $\tau$, at different values of $C_{v}$ (the contribution of $\dot{m}$ is nearly 0 and coincides with the $x$ axis). At solids concentrations by volume lower than 0.10 , the values of the uncertainties in the sluicing time are highest. The overall uncertainty in the sluicing time, which is the root mean square of the sum of all the parametric uncertainties, is shown in Figure 5.6.

The components of the uncertainty, which are caused by errors in the measured mass flow rate and the average slurry density, as obtained by the Micromotion mass flowmeter, are very small. At $\mathrm{C}_{\mathrm{v}}=0.1$, the overall uncertainty in the sluicing time is about three hours, and the main contributions to this error are the uncertainties in the measurements of the carrier liquid and solid particle densities. Without accurate information on these two parameters, the measurements of the mass flow rate and the average slurry density, as obtained from the Micromotion device at low volume concentrations, $C_{v} \leq 0.1$, will always have a high level of uncertainty. This situation can

(a) Crea BA (NHC). March 1997. Electronic communication to CW Stewart and FF Erian (PNNL). 


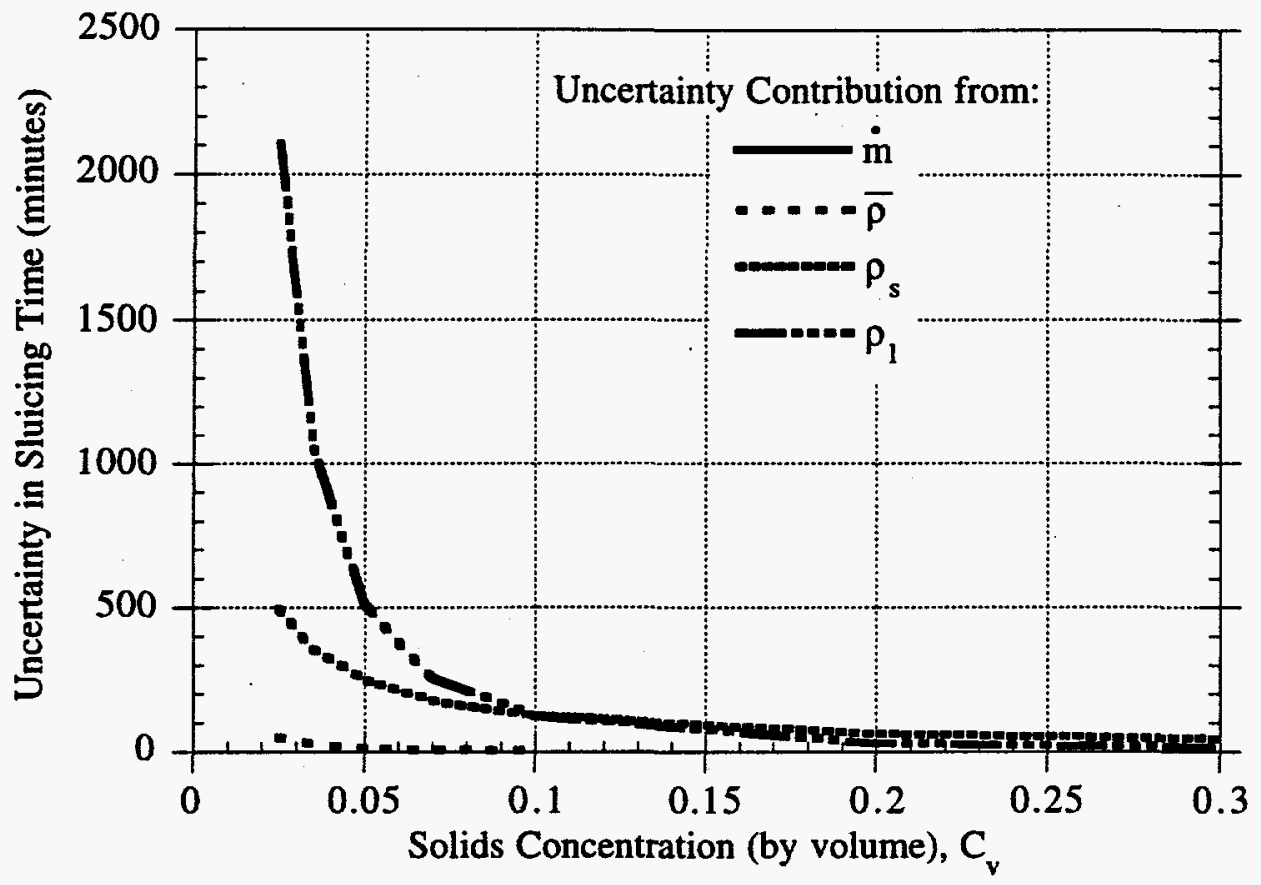

Figure 5.5. Parametric Contributions to Uncertainty in Sluicing Time

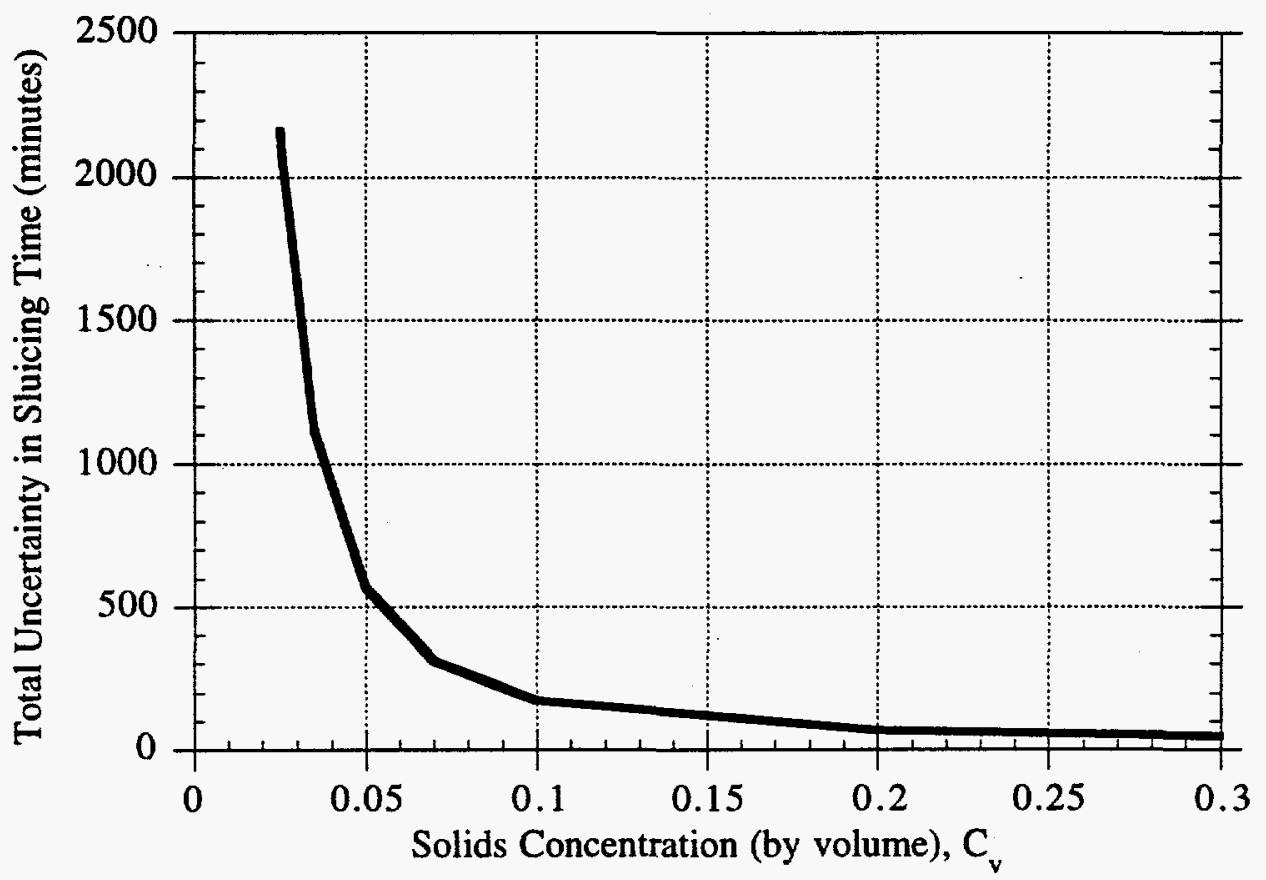

Figure 5.6. Overall Uncertainty in Sluicing Time 
be remedied by ensuring that the sluicing process is efficient and capable of mobilizing the sludge in slurry form at concentrations by volume that are $C_{v}>0.1$.

Another important process control parameter is the solids volume flow rate, $\dot{\mathrm{V}}_{\mathrm{s}}, \mathrm{ft}^{3} / \mathrm{hr}$, which is defined as

$$
\dot{\mathrm{V}}_{\mathrm{s}}=\frac{\dot{\mathrm{m}}}{\bar{\rho}} \mathrm{C}_{\mathrm{v}}, \mathrm{ft}^{3} / \mathrm{hr}
$$

where all symbols have been previously defined except $\dot{\mathrm{V}}_{\mathrm{s}}$, which is the solids volume flow rate. This quantity can be obtained readily from the two outputs of the Micromotion flowmeter after $\mathrm{C}_{\mathrm{v}}$ has been obtained.

The solids volume flow rate, $\dot{\mathrm{V}}_{\mathrm{s}}$, is a more useful process control parameter than the sluicing time, $\tau$, because it gives the solids flux per unit time directly. It also allows us to determine the heat load being transferred from C-106 to AY-102. Based on the parametric uncertainties given in the previous example, the uncertainties in estimating the solids volume flow rate, $\dot{\mathrm{V}}_{\mathrm{s}}$, are shown in Figure 5.7.

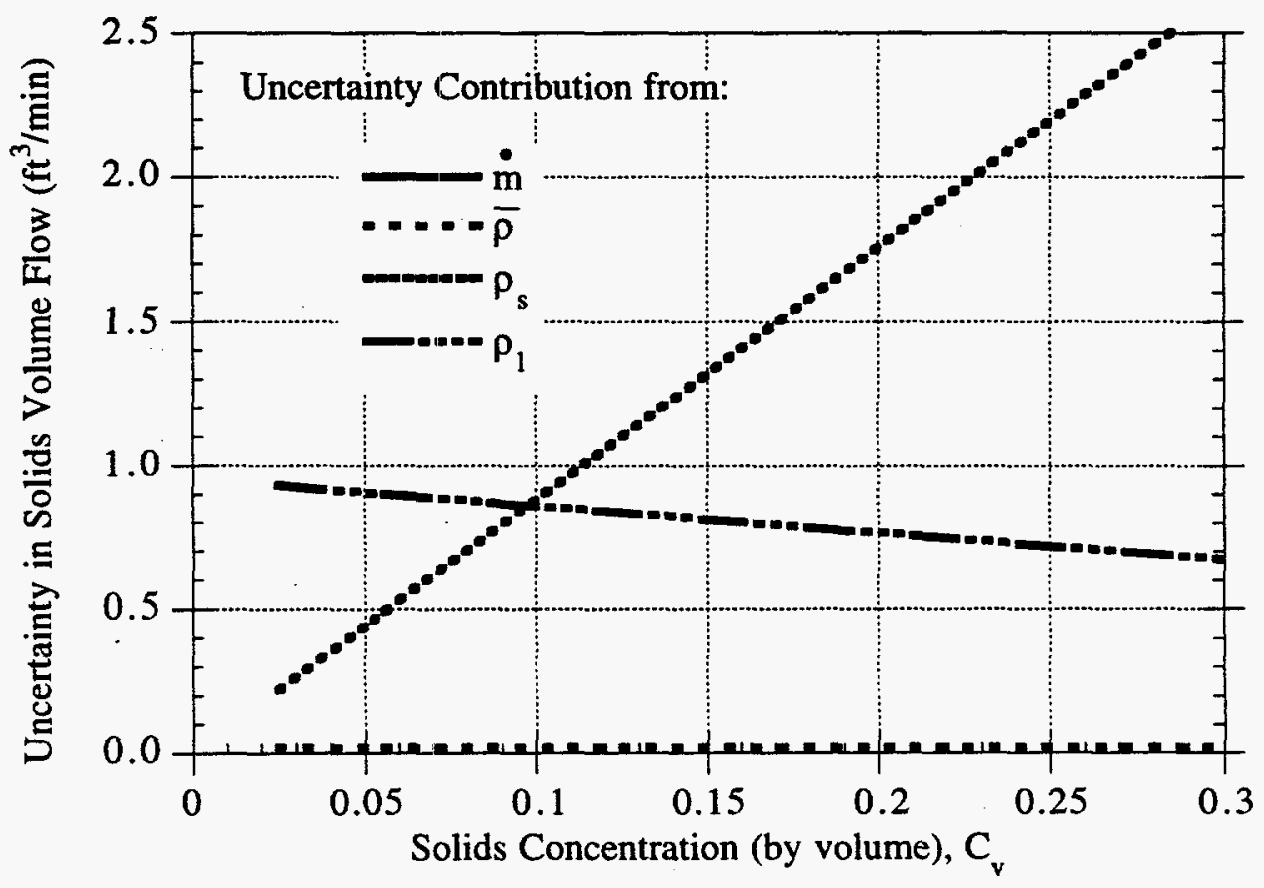

Figure 5.7. Parametric Contributions to Uncertainty in Solids Volume Flow Rate 
Figure 5.7 shows the contributions of the uncertainties in the different parameters that contribute to the estimated solids volume flow rate, $\dot{V}_{s}$, at different values of $C_{v}$ (the contribution of $\dot{m}$ is nearly 0 and coincides with the $x$ axis). At solids concentrations by volume lower than 0.10 , the values of the uncertainties in the solids volume flow rate are lowest. The overall uncertainty in the solid volume flow rate, which is the root mean square of the sum of all the parametric uncertainties, is shown in Figure 5.8. The trend in this figure is opposite the trend shown in Figure 5.6 for the sluicing time. For greater accuracy, it is tempting to use $\dot{\mathrm{V}}_{s}$ as the process control parameter while using the sluicing time as the process control parameter at higher solids concentration by volume. In fact, when one considers the relative or percent error, the two parameters show identical behavior at the various solids concentrations by volume.

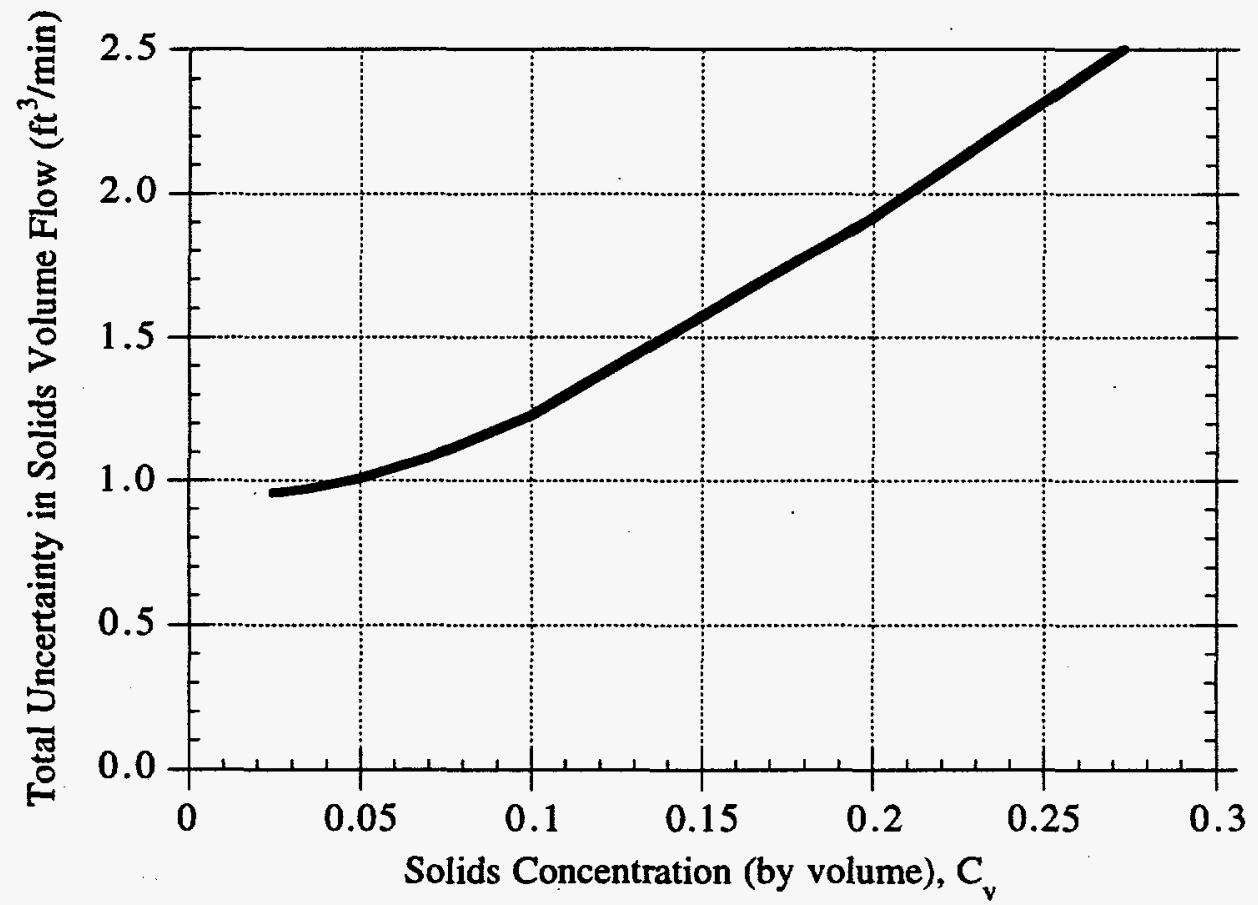

Figure 5.8. Overall Uncertainty in Solids Volume Flow Rate

\subsubsection{Monitoring Heat Load Transfer}

The feasibility of monitoring in real time the heat load associated with the radioactive waste being transferred as a slurry from C-106 to AY-102 has been evaluated in a recent PNNL letter report ${ }^{(a)}$ Two approaches have been studied. The first relies on the detection of radiation from the decay of radioactive elements, and the second uses the detection of heat from the decay of the same elements (calorimetry). Radiation detection is believed to be the most feasible way to estimate the heat load. Two methods for radiation detection have been proposed; both require developmental efforts.

(a) Erian FF and DM Pfund. March 1997. A Feasibility Study for Monitoring Heat Load Transfer Between Tanks 241-C-106 and 241-AY-102. PNNL, Richland, Washington. 
The transfer line is a pipe-in-pipe type construction with a dead air space in between. This geometry ensures that any radioactive slurry leakage from the inner pipe will be trapped in the dead air space by the outer containment pipe. Gamma radiation from the slurry medium flowing in the inner transfer pipe will be partially transmitted through the fluid, the pipe wall, the dead air space between the two pipes, and the containment pipe wall, allowing nonintrusive detection without penetrating the transfer line. Cesium-137 has a strong gamma emission line at $661.6 \mathrm{KeV}$ and thus can be easily detected outside the pipe during transport.

Determining the quantity of ${ }^{90} \mathrm{Sr}$ in the transfer line is more difficult, however, because it is mainly a beta emitter and has no garnma emission. Fortunately, ${ }^{90} \mathrm{Y}$ always exists in equilibrium with its parent isotope, ${ }^{90} \mathrm{Sr}$. Yttrium-90 is also a beta emitter but at considerably higher decay energies; it also yields a very weak (approximately $0.011 \%$ ) ${ }^{90} \mathrm{Zr}$ gamma line at $1.7607 \mathrm{MeV}$. This signal will be undetectable above the background gamma radiation signal and therefore cannot be used as the basis of an assay. However, the bremsstrahlung radiation produced as the beta particles from ${ }^{90} \mathrm{Y}$ are slowed down by collisions in the slurry and by the pipe wall can be detected. in the energy range 1.2 to $2.3 \mathrm{MeV}$ and can be used to identify this high-energy beta decay of ${ }^{90} \mathrm{Y}$ in the pipeline. This energy range is free of interfering emissions from ${ }^{137} \mathrm{Cs}$. Methods based on measuring the bremsstrahlung spectra, or a bremsstrahlung coincidence approach, are currently under development. Acquiring the data is relatively easy, but relating them to actual ${ }^{90} \mathrm{Sr}$ concentration requires a difficult calibration of the counting geometry versus the waste stream composition. The availability of these types of instruments depends on technology development and schedule issues, and it is not a requirement of either the safety assessment or the process control plan. 


\subsection{Data Management Plan}

The recommendations in this section address the management of process control data generated as part of the W-320 Project. The transfer of waste from C-106 to AY-102 requires that several different conditions be monitored to ensure both safe operations and effective results. The conditions in each tank must be monitored as must information about the transfer system. The data must be observable in near real time to support safe operations and to confirm transfer of material. The data must also be stored for later analysis so that heat load and potential flammable gas accumulation issues can be evaluated. There are a number of instruments already available in each tank as well as in the transfer system. All the data from these instruments must be gathered in one location to support the operation and analysis objectives.

\subsection{Data Format and Content}

The data should be maintained in electronic format so it can be stored and accessed via the local area network (LAN). The format should be compatible with existing analytical tools such as Microsoft Excel ${ }^{\circledR}$ to minimize software development cost and promote its use and analysis. The standard format, comma delimited ASCII with standard time stamp information, is essential for this purpose. The data may be stored in current database systems as long as users have free access and the data are easily retrievable for analysis. Data recording schemes that do not record data at a regular frequency but only as it changes should be avoided. Recording relative changes that do not occur on a regular interval requires that they must first be translated into an alternative format.

Any information related to the testing and validation of the instruments should also be made available to analysts but not included in the actual data. These data will help prevent misidentification of trends and events that might otherwise influence the analysis.

Additional supporting information such as operating logs, data recording ranges, sample intervals, error conditions, missing data, and other potential impacts on the data should also be captured and stored with the data. To the extent possible, all these data should be maintained in electronic format for convenient distribution. These meta data will support tracking of events and help identify potential external influences on the data which can then be considered during analysis.

\subsection{Data Handling}

Data from the various instruments should be transferred/transmitted to a network server on the LAN. It should be transferred on a regular schedule so that the most recently recorded data are available for analysis within one day of generation. Since some instruments such as thermocouples need only be recorded daily, this requirement should be adequate for most data. The data may be transmitted via poled modem or direct network access. In either case, adequate backup on the recording device should be provided to ensure minimal loss of data if the normal communication channels malfunction. In some cases, it may be necessary to retrieve data from the recording instrument by visiting the site and retrieving the data to a floppy disk or other recording medium and later placing this information on the server. IF this occurs, the data must be made accessible as soon as possible.

The recorded data must be validated to ensure analytical results can be accepted. Missing data, unphysical spikes, and other errors must be identified and corrected to the extent possible. The raw recorded data will not be modified for any purpose. Missing data that are subsequently 
retrieved will be used to replace gaps in the raw data. Periodic evaluation of the data will help identify potential problems with instruments that can be corrected in the field.

The data should be processed to produce average, minimum, maximum, and standard deviations that can be used to identify anomalous events and for data quality tracking. The processed data will be archived along with the raw recorded data.

\subsection{Data Management Team}

A Data Management Team (DMT) consisting of data users, those responsible for the instrumentation, and data handlers should be established to get the best data to the users as quickly and as conveniently as possible. The team will meet periodically to discuss activities and potential problems related to recording, distribution, validation, and analysis of the data.

The DMT will review the data collected and make recommendations to improve the data quality. The DMT members will be expected to respond to request for information by the team and to make recommendations as it relates to their specific individual responsibilities.

The time and frequency of periodic meetings will be established by the team and will continue as long the project is operational or until the team decides there is no longer any purpose or benefit to continuing.

\subsection{Instrumentation}

A list of instruments that are available or that will be added to the tanks or transfer system must be compiled and maintained. This list should include the manufacturer name and type of instrument, recording range, units, recording frequency, accuracy, recording medium or communication path, special features, testing and validation history, location within the system, installation information, locations of drawings and other visual data, and the names of those who are familiar with and responsible for the instrument.

It is most important to know how the data eventually gets to the server where it is stored and distributed. All this information will help identify potential problems before they can have much influence on the data and to ensure adequate information is being provided to support the required analyses. It is essential that the recorded data reflect both the accuracy and relative precision of the instrument and that these data are adequate for analysis. 


\subsection{References}

Reynolds DA. 1997. Chemical and Chemically Related Considerations Associated with Sluicing Tank-C-106 Waste to Tank AY-102. HNF-SD-WM-TI-756 Rev. 1, Lockheed Martin Hanford Corp., Richland, Washington.

Farnworth SK. 1997. Design Basis Document: Project W-320 Tank 241-C-106 Sluicing Project Rebaselining Change Request New Scope. HNF-SD-W320-DB-001 Rev. 0. Numatec Hanford Corp., Richland, Washington.

Meyer PA, ME Brewster, SA Bryan, G Chen, LR Pederson, CW Stewart, and G Terrones. 1997. Gas Retention and Release Behavior in Hanford Double Shell Waste Tanks. PNNL-11536 Rev. 1, Pacific Northwest National Laboratory, Richland, Washington.

Pasamehmetoglu KO, WL Kubic, and P Sadasivan. 1997. Discussion of Flammable Gas Issues for Project W-320. LA-UR-97-1330 Rev. 0, Los Alamos National Laboratory, Los Alamos, New Mexico.

Sathyanayarana K, DM Ogden, BC Fryer, and MJ Thurgood. 1997. Project W-320 Thermal Evaluation. HNF-SD-W320-ER-002 Rev. 0, Numatec Hanford Corp., Richland, Washington.

Sathyanayarana K. 1996. Evaluation of Potential and Consequences of Steam Bump in High Heat Waste Tanks and Assessment and Validation of the GOTH Computer Code. WHC-SD-WM$\mathrm{CN}-022$ Rev. 0, Westinghouse Hanford Company, Richland, Washington.

Shekarriz A, DR Rector, LA Mahoney, MA Chieda, JM Bates, RE Bauer, NS Cannon, BE Hey, CG Linschooten, FJ Reitz, and ER Siciliano. 1997. Composition and Quantities of Retained Gas Measured in Hanford Waste Tanks 241-AW-101, 241-A-101, 241-AN-105, 241-AN-104, and 241-AN-103. PNNL-11450 Rev. 1, Pacific Northwest National Laboratory, Richland, Washington.

Stewart CW, JM Alzheimer, ME Brewster, G Chen, RE Mendoza, HC Reid, CL Shepard, and G Terrones. 1996a. In Situ Rheology and Gas Volume in Hanford Double-Shell Waste Tanks. PNNL-11296, Pacific Northwest National Laboratory, Richland, Washington.

Stewart CW, ME Brewster, PA Gauglitz, LA Mahoney, PA Meyer, KP Recknagle, and HC Reid. 1996b. Gas Retention and Release Behavior in Hanford Single-Shell Waste Tanks.

PNNL-11391, Pacific Northwest National Laboratory, Richland, Washington.

Whitney PD. 1995. Screening the Hanford Tanks for Trapped Gas. PNL-10821, Pacific Northwest Laboratory, Richland, Washington.

Whitney PD, PA Meyer, NE Wilkins, F Gao, and AG Wood. 1996. Flammable Gas Data Evaluation Progress Report. PNNL-11373, Pacific Northwest National Laboratory, Richland, Washington.

Whyatt GA, RJ Serne, SV Mattigod, Y Onishi, MR Powell, JH Westsik, LM Liljegren, GR Golcar, KP Recknagle, PM Doctor, VG Zhirnov and J Dixon. 1996. Potential for Criticality in Hanford Tanks Resulting from Retrieval of Tank Waste. PNNL-11304, Pacific Northwest National Laboratory, Richland, Washington. 
Wilkins NE, RE Bauer, and DM Ogden. 1996. Results of Vapor Space Monitoring of Flammable Gas Watch List Tanks. HNF-SD-WM-TI-797, Westinghouse Hanford Company, Richland, Washington. 